\title{
Social Choice and Electoral Competition in the General Spatial Model ${ }^{*}$
}

\author{
Jeffrey S. Banks \\ Division of Humanities and Social Sciences \\ California Institute of Technology \\ John Duggan ${ }^{\ddagger}$ \\ Department of Political Science \\ and Department of Economics \\ University of Rochester \\ Michel Le Breton \\ GREMAQ and IDEI \\ Université de Toulouse 1
}

June 28, 2004

\begin{abstract}
This paper extends the theory of the core, the uncovered set, and the related undominated set to a general set of alternatives and an arbitrary measure space of voters. We investigate the properties of social preferences generated by simple games, we extend results on generic emptiness of the core, we prove the general nonemptiness of the uncovered and undominated sets, and we prove the upper hemicontinuity of these correspondences when the voters' preferences are such that the core is nonempty and externally stable. Finally, we give conditions under which the undominated set is lower hemicontinuous.
\end{abstract}

\footnotetext{
* Jeff Banks passed away on December 21, 2000. The second and third authors wish to express their respect and admiration for Jeff as a colleague and dear friend. We will miss him.

${ }^{\dagger}$ We are grateful for discussions with Kim Border, Mark Fey, Cesar Martinelli, Nicholas Miller, and Norman Schofield. We also thank a referee for helpful comments.

${ }^{\ddagger}$ Support from the National Science Foundation, grant number SES-0213738, is gratefully acknowledged.
} 


\section{Introduction}

Since the seminal work of Downs (1957), followed by Davis and Hinich's (1966) introduction of the mathematics of Euclidean preferences, by Plott's (1967) investigation of symmetry conditions for core points, and by Kramer's (1972) adaptation of Farquharson's (1969) analysis of strategic voting in committees, the spatial theory of elections and committees has occupied a prominent theoretical status in the analysis of political decision-making. As pointed out by Ordeshook (1993), "The idea of spatial preferences, of representing the set of feasible alternatives as a subset of an $m$-dimensional Euclidean space, of labelling the dimensions 'issues,' of assuming that people (legislators or voters) have an ideal policy on each issue, and of supposing that each person's preference (utility) decreases as we move away from his or her $m$-dimensional ideal policy, is now commonplace and broadly accepted as a legitimate basis for modelling electorates and parliaments." ${ }^{1}$ The classical approach to the analysis of the spatial model takes as given the set of alternatives and voter preferences over them, and it formalizes the decisions of coalitions of voters in terms of a dominance, or "social preference," relation, which captures the incentives of groups to form and move from one alternative to another. This relation reflects not only voter preferences, but also institutional features that may favor some groups over others, such as voting rights laws, laws governing campaign contributions, or the partitioning of voters into districts. Thus, implicit in this level of abstraction is the assumption that it is not necessary to model the precise details of such political institutions.

The maximal elements of the dominance relation, known as the "core," occupy the special position of being socially at least as good as all other alternatives, but it is well known that the majority core may be empty. Indeed, building on the work of Plott (1967), a number of authors have established that emptiness of the core is a generic property in multidimensional settings, where "generic" is defined formally in various ways. ${ }^{2}$ Furthermore, McKelvey's $(1976,1979)$ "chaos" results showed that emptiness of the core typically leads to a social preference cycle throughout the space of alternatives. Some scholars (e.g., Riker (1980)) have concluded from this that political decisions represent arbitrary outcomes highly dependent upon the specific details of the particular institutions under consideration, and, therefore, that a general theory of political decision-making is impossible. This point of view has been challenged by others searching for "institution-free" properties of social choice to provide bounds on equilibrium predictions and to circumscribe the extent of instability. The problem, abstractly formulated, is to construct a compelling theory of choice that is consistent with maximality when the core is nonempty but yields nonempty choice sets even when the core does not.

In that vein of research, the notion of the uncovered set is central. The uncovered set was defined originally by Fishburn (1977) and Miller (1980) and axiomatized by Moulin (1986) in the context of tournaments, i.e., majority preferences over a finite set of alternatives

\footnotetext{
${ }^{1}$ See Austen-Smith and Banks (1999) for background on the spatial model of social choice.

${ }^{2}$ These include Rubinstein (1979), Schofield (1983), Cox (1984), Le Breton (1987), Banks (1995), and Saari (1997).
} 
with no ties. ${ }^{3}$ In that setting, one of several equivalent definitions of the uncovered set is as follows: an alternative $x$ belongs to the uncovered set if and only if, for every alternative $z$, there is some $y$ such that $x$ is socially preferred to $y$, which is socially preferred to $z$. The comparison with the core is clear: rather than requiring that $x$ be directly preferred to every other alternative, we allow it to be indirectly preferred in two steps. The uncovered set can also be viewed as the maximal elements of a transitive "covering relation," and it follows immediately that the uncovered set is nonempty when the set of alternatives is finite. In the corresponding model of two-party electoral competition, based on Downs (1957), two parties independently adopt policy positions in the space of alternatives, with the winner being the party adopting the socially preferred position. It is well known that a pair of policy positions form a pure strategy Nash equilibrium if and only if the parties both locate at core points. In case the core is empty, existence of a mixed strategy equilibrium is not problematic in the finite model. Fisher and Ryan (1992) and Laffond, Laslier, and Le Breton (1993) independently proved that the Downsian electoral game has a unique mixed strategy equilibrium, and that the support of the parties' strategies lies in the uncovered set. In the context of weak tournaments, where majority ties are allowed, distinctions emerge between previously equivalent definitions of the uncovered set. We focus on the uncovered set, as defined by McKelvey (1986) and several later authors, ${ }^{4}$ and on the smaller "undominated set." While the undominated set is too small to bound mixed strategy equilibria in the Downsian electoral game, Dutta and Laslier (1999) show that the uncovered set still contains the support of all equilibrium mixed strategies.

Shepsle and Weingast (1984) and McKelvey (1986) were the first to consider the uncovered set in the standard spatial model, where policy alternatives are modelled as points in a convex subset of Euclidean space and majority preferences over social policies are determined by the continuous, strictly convex preferences of a finite electorate. Under these assumptions, the covering relation is still transitive, but its continuity properties are poor, complicating the problem of existence of an uncovered alternative. Nevertheless, McKelvey demonstrates that the uncovered set (indeed, the undominated set as well) is nonempty and, under the stronger assumption that preferences are Euclidean, he shows that this set lies in a centrally located region of the policy space, one that collapses to the core as voter preferences are aligned to make the core nonempty. This result is generalized by Cox (1987) to strictly convex voter preferences exhibiting "limited asymmetry." 5 A more general result on the nonemptiness of the uncovered set is derived by Bordes, Le Breton, and Salles (1992), with a yet more general result proved by Banks, Duggan, and Le Breton (2002). An important contribution of McKelvey's (1986) is the observation that the uncovered set bounds equilibrium outcomes in several different institutional settings, including sophisticated voting outcomes for a class of binary trees and mixed strategy equilibria of the

\footnotetext{
${ }^{3}$ See Laslier (1997) for a comprehensive reference on tournaments.

${ }^{4}$ See Dutta and Laslier (1999), Peris and Subiza (1999), Duggan, and Le Breton (2001), and Banks, Duggan and Le Breton (2002).

${ }^{5}$ The precise calculation of the uncovered set, or, more modestly, the search for bounds sharpening those discovered by McKelvey in some specific situations, is the subject of Feld, Grofman, Hartley, Kilgour, and Miller (1987) and Hartley and Kilgour (1987).
} 
associated Downsian electoral game. His claim for the latter setting is formally proved and extended by Banks, Duggan, and Le Breton (2002).

The main objective of this paper is to contribute further to this research program by developing the theory of the core, the uncovered set, and the undominated set in very general environments. We begin in an abstract choice theory setting with a topological space of alternatives and an arbitrary preference relation; we establish weak sufficient conditions for nonemptiness of the uncovered set and the undominated set; and we examine other properties of these sets, such as external stability and the possibility of a "two-step" principle. We then explicitly model an electorate as a measure space of voters and a profile of preferences assigning each voter a continuous weak order on the space of alternatives. Thus, we discard the usual assumptions on the set of alternatives and voter preferences and instead impose general topological conditions on these primitives, allowing us to capture, for example, the choice of an income tax function or the choice of a probability measure over an infinite set. In doing so, we unify the cases of a finite set of alternatives and the spatial model, previously treated only separately. Further, by dropping the common assumption of strictly convex preferences, we capture a number of common models in economics and political science: we obtain as special cases private good and mixed economies, with or without production, as well as the "divide-the-dollar" model, where voters must allocate a fixed amount of a resource among themselves and care only about their own consumption. ${ }^{6}$

Our assumption of an arbitrary measure space of voters captures a finite electorate and a continuous distribution of voters as special cases. For example, in the spatial model with preferences parameterized by "ideal points," say in $\Re^{n}$, we may describe the electorate either by a finite number of points in $\Re^{n}$, as in the simplest model, or by a density with respect to the Lebesgue measure. In the context of an exchange economy, we may assume a finite number of consumers or, as in Hildenbrand (1974), allow for arbitrary distributions of preferences over net consumption bundles. In fact, though empirical preference distributions are discrete, a substantial literature on voting models them as continuous. For example, Downs (1957) and Tullock (1967) have discussed, in very unformalized terms, the existence of equilibria for continuous voter distributions. In the Euclidean setting, Davis, Degroot, and Hinich (1972) allowed for a continuum of voters and found that the existence of majority equilibrium was equivalent to the existence of a total median in the distribution of ideal points. Kramer (1978) proved the existence of mixed strategy Nash equilibria in the multidimensional Downsian model, assuming a continuous distribution of voters and vote-maximizing parties. ${ }^{7}$ McKelvey, Ordeshook, and Ungar (1980) have extended Plott's necessary symmetry condition for core points to the case of a measure space of voters. Myerson (1993) examines Nash equilibria in electoral competition between parties that must compete for votes by, essentially, dividing a dollar across a continuous electorate.

There are several reasons why it is desirable to model electorates at this level of general-

\footnotetext{
${ }^{6}$ In these environments, an agent's preferences may be strictly convex in his own consumption but not in others'. Since an alternative must specify the consumption of all consumers, strict convexity is not satisfied.

${ }^{7}$ Duggan (2002) provides a simplified proof. Duggan (2002) and Duggan and Jackson (2004) analyze the issue of existence of mixed strategy equilibrium with a finite number of voters.
} 
ity. First, regularity conditions across voter preferences may be more easily formalized and analyzed. This type of concern is evident in the work of Grandmont (1978) on intermediate preferences and in the work of Caplin and Nalebuff (1988), who have shown in the Euclidean setting that, if a continuous voter distribution is described by a log-concave density and we increase the majority quota to $64 \%$ or more, then there exists an undefeated policy. Second, the continuous setting is an important step toward our understanding of finite but large electorates. When we deal with a finite electorate, we do not usually explain how the diversity of preferences among the electorate is generated, i.e., we simply consider as given a finite list of points in the relevant space of preferences. But suppose that the finite list of preferences in the electorate is a sample of independent draws from an underlying continuous distribution on preferences. Then, if the electorate is large, the Glivenko-Cantelli Theorem implies that the continuous distribution is a good "approximation" of the finite one. Consequently, if continuity results can be established on some sets, e.g., the uncovered set, then the sets defined for the limit distribution will "approximate" the sets for large, finite electorates. Third, if elections, rather than committees, are the main object of study, then it is desirable that results not be too sensitive to specific assumptions about whether the number of voters is odd or even. This means that adding or deleting one voter should not matter, so that each voter is essentially massless, which leads us to the continuum model. Finally, in case alternatives are ultimately chosen as the result of Downsian competition between two parties, we have existence of mixed strategy equilibrium in models with a continuum of voters, as in Kramer (1978), whereas the problem of existence remains unsolved for finite electorates: the presence of a continuum of voters helps to "smooth out" the parties' payoffs, eliminating certain discontinuities that are extremely problematic in the finite case.

While the above authors consider majority rule only or restrict attention to anonymous voting rules, we build on the concept of a simple game to describe the distribution of power in a large electorate. Our definition extends Shapley's (1962) notion of simple game, defined for a finite set of players, to a measure space of voters, but we add the restriction that measure zero sets of voters "don't count," an intuitive idea in a model of political decisionmaking in a democratic society. We explore the implications of this new structure, and we define several new continuity properties of simple games that generalize majority rule and play an important role subsequently. We then analyze the properties of social preferences induced by simple games. Notably, we introduce a dispersion condition on voter preferences that plays a role analogous to that of an odd number of voters in the finite context: our condition essentially demands that, given any three non-collinear alternatives and any of the six possible rankings of them, the set of voters whose preferences conform to that ranking has positive measure. We use this condition, which holds, for example, if voters have Euclidean preferences and ideal points are distributed according to a density function with full support, to prove that social "indifference curves" are nowhere dense. Finally, we consider the core, the uncovered set, and the undominated set generated by arbitrary electorates. We first prove the generic emptiness of the core, extending earlier results for finite electorates, and we then exploit our choice-theoretic results on the uncovered set and 
undominated set. We show, for example, that if voters have continuous preferences with compact upper sections, and if the simple game satisfies a minimal continuity property, then the uncovered set is nonempty. We also establish, under slightly stronger conditions involving the lower hemicontinuity of upper sections of the social preference relation, the nonemptiness of the undominated set. We show that these sets are upper hemicontinuous at preference profiles where the core is nonempty and externally stable, generalizing Cox's (1987) continuity result. Finally, we give conditions under which the undominated set is lower hemicontinuous, a key condition for the existence of continuous selections, via Michael's Selection Theorem.

Returning to the Downsian electoral model, a policy position is not weakly dominated for a party if and only if it lies in the undominated set, so our result on nonemptiness of the undominated set implies the existence of undominated strategies for the parties, despite the substantial discontinuities in the parties' payoff functions. An implication of our result on the generic emptiness of the core, however, is that, even with a continuum of voters, almost all assignments of preferences to voters lead to the non-existence of pure strategy equilibrium. If there is a mixed strategy equilibrium with probability-of-winning maximizing parties, then Banks, Duggan, and Le Breton (2002) show that the support of all such mixed strategy equilibria must lie in the uncovered set. Our continuity results then have the following implications. Suppose that there is a continuum of voters, and consider a preference profile for which the core is nonempty and externally stable. For example, in the Euclidean setting, suppose voter ideal points are distributed according to a radially symmetric density function. There is then a pure strategy equilibrium, the unique core point, which coincides with the undominated set and uncovered set. Now perturb the distribution of ideal points to violate symmetry, so that the core is empty and pure strategy equilibria no longer exist. By upper hemicontinuity, the uncovered set cannot expand discontinuously to contain points far away from the core of the original profile, and, since the undominated strategies form a subset of the uncovered set, the same is true for them. Mixed strategy equilibria, if any, will have supports contained in the uncovered set, and it follows that these equilibrium strategies must put probability close to one on points nearby the pure strategy equilibria of the original game. Thus, we obtain a robustness result for Downsian elections.

Furthermore, our upper hemicontinuity result can be applied to investigate the properties of the uncovered set for large but finite electorates. Suppose, for example, that the set of alternatives is multidimensional and voter preferences are Euclidean, and consider a distribution over the set of alternatives possessing a unique median in all directions. If we randomly draw voter ideal points from this distribution, then with probability one the core will be empty after any finite number of draws, but the uncovered sets of these finite electorates will be nonempty. Moreover, by the Glivenko-Cantelli Theorem, the empirical distribution of preferences in the finite electorates will approximate the underlying distribution with probability one. After mapping these finite electorates into a model with a fixed, infinite set of voters, our continuity result implies that the corresponding uncovered sets must become arbitrarily close to the core of the underlying distribution, which is the 
median in all directions. It follows that the undominated strategies and the mixed strategy equilibrium outcomes of Downsian competition also converge to this underlying core point. Thus, we confirm a longstanding conjecture by Nicholas Miller that the uncovered set collapses to a centrally located area as the number of voters increases. ${ }^{8}$

The problems we take up in this paper are quite different from another literature on social choice with infinite electorates, which takes an axiomatic approach in the spirit of Arrow (1963). Fishburn (1970), Kirman and Sondermann (1972), and Armstrong (1980), for example, extend Arrow's Theorem to infinite sets of voters without the added structure of a measure on the set of voters. Recently, Gomberg, Martinelli, and Torres (2002) and Fey (2002) have considered the implications of anonymity for a measure space of voters. These authors begin with axioms on aggregation rules defined on a domain of preferences and characterize the rules satisfying these axioms. In contrast, we begin with a restricted class of aggregations rules, and we deduce properties of social preferences generated by a fixed profile of preferences or we deduce properties of certain sets of preference profiles.

In Section 2, we analyze the core, uncovered set, and undominated set in the abstract choice theory setting. In Section 3, we introduce a measure space of voters and define our concept of a simple game. In Section 4, we examine the properties of social preferences derived from an electorate. In Section 5, we present our genericity and continuity results on the core, uncovered set, and undominated set. An appendix contains a general analysis of binary relations, proofs of lemmas, and proofs of the propositions omitted from the text.

\section{Choice Sets}

We consider an abstract setting in this section, letting $P$ be a strict preference relation and $R$ a weak preference relation over a topological space $A$ of alternatives. When we discuss the case of finite $A$, we will always assume the discrete topology. We assume here that $P$ is irreflexive, that $R$ is reflexive, and that the relations are dual: $a R b$ if and only if not $b P a$. Note that $P$ is asymmetric if and only if $R$ is complete, in which case $P$ is the asymmetric part of $R .^{9}$ We say $P$ is a tournament if it is also connected, in the sense that $a \neq b$ implies $a P b$ or $b P a$. Given an arbitrary relation $Q$ on $A$, we denote by $Q(a)$ the set $\{b \in A: b Q a\}$ and by $Q^{-1}(a)$ the set $\{b \in A: a Q b\}$. Note that, by duality, $P^{-1}(a)$ is open if and only if $R(a)$ is closed. An alternative $a$ is $Q$-maximal if, for all $b \in A, b Q a$ implies $a Q b$. If $Q$ is asymmetric, this is equivalent to $Q(a)=\emptyset$; if $Q$ is complete, this is equivalent to $Q^{-1}(a)=X$. For now, we abstract from the details of $P, R$, and $A$, though later $A$ will be given the interpretation of a policy space and $P$ and $R$ will represent social preferences, derived from an explicit collection of "winning coalitions."

A central concept in what follows is the core, denoted $K$, which is defined as the set of

\footnotetext{
${ }^{8}$ See Shepsle and Weingast's (1984) footnote 15. This result is also related to work on convergence of the yolk by Feld, Grofman, and Miller (1988), Koehler (1990), and Tovey (1992) in the spatial model with Euclidean preferences.

${ }^{9}$ The conditions of asymmetry and completeness are standard, but they are used in this section for only two results. We state our other results without those conditions, to maximize their generality.
} 
$P$-maximal (equivalently, $R$-maximal) alternatives. That is, an alternative lies in the core if and only if there is no other alternative strictly preferred to it (equivalently, it is weakly preferred to all other alternatives). When $P$ is asymmetric, as in most applications, we have

$$
K=\{a \in A: P(a)=\emptyset\} .
$$

We define the dominance relation, denoted $D$, as follows: $a D b$ if and only if $P(a) \subseteq P(b)$ and $R(a) \subseteq R(b)$, at least one inclusion strict. The undominated set, denoted $U D$, consists of the $D$-maximal alternatives. Since $D$ is asymmetric, this can be written as

$$
U D=\{a \in A: D(a)=\emptyset\}
$$

Define the covering relation, denoted $C$, as follows: $a C b$ if and only if $a P b, P(a) \subseteq P(b)$, and $R(a) \subseteq R(b)$. Equivalently, $a C b$ if and only if $a P b$ and $a D b$. The uncovered set, denoted $U C$, consists of the $C$-maximal alternatives. Since $C$ is asymmetric, this can be written as

$$
U C=\{a \in A: C(a)=\emptyset\} .
$$

It is clear from these definitions that $K \cup U D \subseteq U C{ }^{10}$

The next proposition gives a condition on preferences sufficient for external stability of the core and for the nesting of the core and undominated set. This condition, that $R(a)=\overline{P(a)} \cup\{a\}$ for every alternative, ${ }^{11}$ formalizes the idea that the "indifference curve" through $a$ is "thin." Indeed, define the relation $I$ as follows: $a I b$ if and only if $a R b$ and $b R a$. An implication of our condition is that, if $P(a)$ is open, then $I(a)$ is nowhere dense. We append the singleton $\{a\}$ to $\overline{P(a)}$ to capture the case in which $A$ is finite and $P$ is a tournament.

Proposition 1 Assume $R(a)=\overline{P(a)} \cup\{a\}$ for all $a \in A$. For all $a \in K$ and all $b \in A \backslash\{a\}$, $a P b$. If $K$ is nonempty, then it is a singleton, and $K=U D=U C$.

Proof: Take any $a \in K$ and any distinct $b \in A$. If not $a P b$, then $b \in R(a)=\overline{P(a)} \cup\{a\}$. Since $b \neq a$, we have $P(a) \neq \emptyset$, contradicting $a \in K$. Thus, $a P b$. It follows that $a C b$ for all $b \in A \backslash\{a\}$, and since $C$ is asymmetric, this implies $K=U D=U C$.

Though $K$ can be empty in the absence of acyclicity or semi-convexity of $P,{ }^{12}$ asymmetry and transitivity of $C$ and $D$ immediately imply nonemptiness of the sets $U D$ and $U C$ when $A$ is finite. Our general results on nonemptiness and external stability of the above sets follow from the analysis of maximal elements in the appendix.

\footnotetext{
${ }^{10}$ Some authors define covering without $P(a) \subseteq P(b)$, and others drop $R(a) \subseteq R(b)$. We show that, in some environments, these omissions are inconsequential. In general, however, both inclusions are needed for the logical nesting of the undominated and uncovered sets.

${ }^{11}$ Given a set $X, \bar{X}$ denotes the closure of $X, X^{\circ}$ denotes the interior, and $X^{c}$ denotes the complement.

${ }^{12}$ We say $P$ is semi-convex if, for all $a \in A, a$ is not contained in the convex hull of $P(a)$. See Austen-Smith and Banks (1999) for a reference on existence of maximal elements under semi-convexity.
} 
Proposition 2 Assume $R(a)$ is compact for some $a \in A$, and $R(b)$ is closed for all $b \in A$. Then $U C \neq \emptyset$.

The nonemptiness of the undominated set is obtained under stronger assumptions on preferences.

Proposition 3 Assume $R(a)$ is compact for some $a \in A, R(b)$ is closed for all $b \in A$, and $R(\cdot)$ is lower hemicontinuous as a correspondence. Then $U D \neq \emptyset$.

We now establish that, under the assumptions of Proposition 3, the undominated and uncovered sets are externally stable.

Proposition 4 Assume $R(a)$ is compact for all $a \in A$ and $R(\cdot)$ is lower hemicontinuous as a correspondence.

1. If $a \notin U D$, then there exists $b \in U D$ such that $b D a$.

2. If $a \notin U C$, then there exists $b \in U C$ such that $b C a$.

We next give conditions that can be used to simplify the definitions of the dominance and covering relations. One condition is "thin indifference curves," i.e., $R(a)=\overline{P(a)} \cup\{a\}$, and the other requires that sections of the strict preference relation "fill" sections of weak preference: $P(a) \cup\{a\}=R(a)^{\circ} \cup\{a\}$. Again, we append the singleton $\{a\}$ to capture the case where $A$ is finite and $P$ is a tournament. The next lemma, which generalizes Shepsle and Weingast's (1984) Lemma 1, does the bulk of the work for us.

\section{Lemma 1}

1. If $R(a)=\overline{P(a)} \cup\{a\}$ and $R(b)=\overline{P(b)} \cup\{b\}$, then $P(a) \subseteq P(b)$ implies $R(a) \subseteq R(b)$.

2. If $P(a) \cup\{a\}=R(a)^{\circ} \cup\{a\}$ and $P(b) \cup\{b\}=R(b)^{\circ} \cup\{b\}$, then $R(a) \subseteq R(b)$ implies $P(a) \subseteq P(b)$.

Before continuing, we note an alternative formulation of the "full weak sections" condition in the second part of Lemma 1. It is used in several results to follow.

Lemma 2 Assume $A$ is Hausdorff. Then $P(a) \cup\{a\}=R(a)^{\circ} \cup\{a\}$ for all $a \in A$ if and only if $R^{-1}(a)=\overline{P^{-1}(a)} \cup\{a\}$ for all $a \in A$.

We now consider some equivalent formulations of the covering and dominance relations. A version of the following proposition can be found in McKelvey's (1986) Proposition 3.3, where he assumes conditions on voter preferences to obtain needed conditions on social

preferences, namely, thin indifference curves and full weak sections. His assumptions are 
indeed sufficient for thin indifference curves, used in part 1 of Proposition 5, below, and so the two equivalences there do hold in his model. McKelvey's assumptions on voter preferences do not, however, entail full weak sections, used in part 2 of the proposition: the condition will hold under his other assumptions if the set $A$ of alternatives is an open subset of $\Re^{n}$, but not generally. The condition is crucial, for without it, the implication

$$
a P b \text { and } R(a) \subseteq R(b) \Rightarrow a C b
$$

may fail. In Section 4, we give two examples of social choice environments, including the divide-the-dollar environment, where this failure occurs. Thus, violations of the full weak sections condition necessitate care in the definition of covering and raise the possibility of multiple "uncovered sets." Note that the conditions of our proposition hold if $A$ is finite and $P$ is a tournament, though the equivalences are well-known in that environment.

\section{Proposition 5}

1. If $R(a)=\overline{P(a)} \cup\{a\}$ for all $a \in A$, then

$$
\begin{aligned}
a C b & \Leftrightarrow a P b \text { and } P(a) \subseteq P(b) \\
a D b & \Leftrightarrow P(a) \subset \subset P(b) .
\end{aligned}
$$

2. Assume $A$ is Hausdorff. If $R^{-1}(a)=\overline{P^{-1}(a)} \cup\{a\}$ for all $a \in A$, then

$$
\begin{aligned}
a C b & \Leftrightarrow a P b \text { and } R(a) \subseteq R(b) \\
a D b & \Leftrightarrow R(a) \subset \subset R(b) .
\end{aligned}
$$

Proof: To prove the first part of the proposition, suppose $R(a)=\overline{P(a)} \cup\{a\}$ for all $a \in A$. Clearly, $a C b$ implies $a P b$ and $P(a) \subseteq P(b)$. For the opposite direction, note that, by Lemma 1, $P(a) \subseteq P(b)$ implies $R(a) \subseteq R(b)$, as required. Clearly, aDb implies $P(a) \subseteq P(b)$. If this inclusion is not strict, then $P(a)=P(b)$, and Lemma 1 implies $R(a)=R(b)$, a contradiction. Therefore, $P(a) \subset \subset P(b)$. For the opposite direction, note that, by Lemma $1, P(a) \subset \subset P(b)$ implies $R(a) \subseteq R(b)$. To prove the second part of the proposition, suppose $R^{-1}(a)=\overline{P^{-1}(a)} \cup\{a\}$ for all $a \in A$. By Lemma 2, we have $P(a) \cup\{a\}=R(a)^{\circ} \cup\{a\}$ for all $a \in A$. Clearly, $a C b$ implies $a P b$ and $R(a) \subseteq R(b)$. For the opposite direction, note that, by Lemma $1, R(a) \subseteq R(b)$ implies $P(a) \subseteq P(b)$, as required. Clearly, $a D b$ implies $R(a) \subseteq R(b)$. If this inclusion is not strict, then $R(a)=R(b)$, and Lemma 1 implies $P(a)=P(b)$, a contradiction. Therefore, $R(a) \subset \subset R(b)$. For the opposite direction, note that, by Lemma $1, R(a) \subset \subset R(b)$ implies $P(a) \subseteq P(b)$.

We end this section by considering the possibility of deriving a version of the "two-step" principle (Miller (1980)) in our abstract setting. We first prove a simple lemma, a version of which can be found in McKelvey's (1986) Proposition 3.4. ${ }^{13}$ For a binary relation $Q$ on $A$, we denote by $Q^{2}(a)$ the set $Q(a) \cup\{b \in A: \exists c \in A, b Q c Q a\}$.

\footnotetext{
${ }^{13}$ Part 1 of Lemma 3 generalizes Shepsle and Weingast's (1984) lemma 2(b); part 2 of Lemma 3 generalizes their Lemma 5; and part 3 of Lemma 3 generalizes their Lemma 2(a). In the latter result, however, the authors omit the antecedent condition of part 3, which, as we show in Section 4, is needed for the result.
} 
Lemma 3 Assume $R$ is complete and $P$ is asymmetric.

1. If $a \in P^{2}(b)$, then $P(b) \nsubseteq P(a)$ and $R(b) \nsubseteq \subset R(a)$.

2. If $P(b) \nsubseteq P(a)$ or $R(b) \nsubseteq R(a)$, then $a \in R^{2}(b)$.

3. If $R^{-1}(a)=\overline{P^{-1}(a)} \cup\{a\}$ for all $a \in A$, then $a \in P^{2}(b)$ if and only if $P(b) \nsubseteq \perp P(a)$.

4. If $R(a)=\overline{P(a)} \cup\{a\}$ for all $a \in A$, then $a \in P^{2}(b)$ if and only if $R(b) \nsubseteq R(a)$.

We now state a form of the two-step principle. The first part of Proposition 6 is as in Shepsle and Weingast's (1984) Proposition 2 and McKelvey's (1986) Proposition 4.1. In the latter paper, however, McKelvey also states that $U C$ is contained in the closure of $\bigcap_{a \in A}\left(P^{2}(a) \cup\{a\}\right),{ }^{14}$ but his proof contains an error, ${ }^{15}$ and we have not been able to verify the result. Thus, the size of the gap between the uncovered and undominated sets, despite the similarities in their definitions, remains open. The next result does establish, under a richness condition on the strict preference relation, a strong version of the two-step property for the undominated set: given any undominated alternative $a$ and any other $b$, either $a$ is strictly preferred to $b$, or it is strictly preferred to some alternative that is itself strictly preferred to $b$.

Proposition 6 Assume $R$ is complete and $P$ is asymmetric.

1. $\bigcap_{a \in A}\left(P^{2}(a) \cup\{a\}\right) \subseteq U D \subseteq U C \subseteq \bigcap_{a \in A} R^{2}(a)$.

2. Assume $R^{-1}(a)=\overline{P^{-1}(a)} \cup\{a\}$ and $R(a)=\overline{P(a)} \cup\{a\}$ for all $a \in A$, and $P$ is open. If $a \in U D$ and $b \notin U D$, then $a \in P^{2}(b)$. If $a \in U C$ and $b \notin U C$, then $a \in P^{2}(b)$.

3. Moreover, under the latter assumptions, if $a \in U D$ and $b \in A \backslash\{a\}$, then either $a \in P^{2}(b)$ or $P(a)=P(b)$.

Proof: That $\bigcap_{a \in A}\left(P^{2}(a) \cup\{a\}\right) \subseteq U D$ follows from the first part of Lemma 3 . That $U C \subseteq \bigcap_{a \in A} R^{2}(a)$ follows from the second part of Lemma 3. To prove the second part of the proposition, take $a \in U D$ and $b \notin U D$. Let $c D b$. Since not $c D a$, either $P(c) \nsubseteq P(a)$, or $R(c) \nsubseteq R(a)$, or both $P(c)=P(a)$ and $R(c)=R(a)$. In the first two cases, the third and fourth parts of Lemma 3 yield $a \in P^{2}(c)$ and, therefore, $a \in P^{2}(b)$. In the last case, $a D b$, and at least one of $P(a) \subseteq P(b)$ and $R(a) \subseteq R(b)$ holds strictly, and Lemma 3 again implies $a \in P^{2}(b)$. Now take $a \in U C$ and $b \notin U C$. Let $c C b$. Since not $c C a$, there are four possible cases: the three above, which proceed as before, and $a R c$. If $a=c$, then, since $c P b$, we are done. If $a \neq c$, then $c \in \overline{P^{-1}(a)}$. Since $c \in P(b)$, an open set, there exists $d \in P^{-1}(a) \cap P(b)$, which means $a \in P^{2}(b)$. To prove the third part of the proposition, take $a \in U D$ and $b \neq a$. Then either $P(b) \nsubseteq P(a)$ or $R(b) \nsubseteq R(a)$ or $P(b)=P(b)$. By parts 3 and 4 of Lemma 3 , each of the first two cases imply $a \in P^{2}(b)$.

\footnotetext{
${ }^{14}$ After correcting a typo: the set $\bigcap_{a \in A} P^{2}(a)$ considered by McKelvey is necessarily empty, by asymmetry of $P$. Shepsle and Weingast consider the same set.

${ }^{15}$ In the last line on p.309, McKelvey claims that " $\bigcap_{y \in X} \overline{P^{2}(y)} \subseteq \overline{\bigcap_{y \in X} P^{2}(y)}$," which need not hold.
} 
The results of this section have been presented in the framework of abstract choice theory, but their main application concerns collective choice situations, where $P$ and $R$ aggregate the preferences of a group of voters. The next section provides a general model of the set of voters and the decision-making power of coalitions.

\section{Electorates}

The purpose of this section is to introduce a general framework, describing an electorate as a measurable mapping from an abstract set of voters into the set of continuous weak orderings of alternatives. The framework is general enough to accommodate a finite number or a continuous distribution of voters. An electorate consists of a probability space $(\Omega, \Sigma, \lambda)$, where $\Omega$ is a set of voters (or voter "types"), a $\sigma$-algebra $\Sigma$ on $\Omega$, and a probability measure $\lambda$, together with a preference profile $\rho$, formalized as follows. Let $A$ denote a topological space of alternatives, and let $\mathcal{R}$ denote the set of closed weak orders on $A$ (complete, transitive relations, closed in $A \times A$ ), endowed with the topology of closed convergence (Hildenbrand (1974)). Endowing $\mathcal{R}$ with the Borel $\sigma$-algebra, a profile is a measurable mapping $\rho: \Omega \rightarrow \mathcal{R}$, where $\rho(\omega)$ is the weak preference relation of voter $\omega$. Let $\pi(\omega)$ denote the asymmetric part of $\rho(\omega)$, the strict preference relation of voter $\omega$. If $A$ is a Hausdorff, locally compact space, then, given any $a, b \in A$, the set $\{R \in \mathcal{R}: a R b\}$ is closed in the topology of closed convergence, and, since $\rho$ is measurable, the coalitions $\{\omega \in \Omega: a \rho(\omega) b\}$ and $\{\omega \in \Omega: a \pi(\omega) b\}$ are $\Sigma$-measurable. If $\Omega$ is a topological space, in which case we assume that $\Sigma$ consists of the Borel sets, and if $\rho$ is continuous, then these coalitions of voters are closed and open, respectively. Except for the regularity imposed by measurability, the notion of electorate is simply the direct extension of the notion of profile used when there is a finite number of voters.

We now turn to our formal representation of the distribution of power in the electorate, a concept that underlies our analysis of social preferences in the next section.

Definition 1 A simple game is a collection $\mathcal{W} \subseteq \Sigma$ of coalitions such that $\emptyset \notin \mathcal{W}, \Omega \in \mathcal{W}$, and, for all $S \in \overline{\mathcal{W} \text { and all } T} \in \Sigma, \lambda(S \backslash T)=0$ implies $T \in \mathcal{W}$.

The coalitions in $\mathcal{W}$ are winning coalitions. Note that, by our definition, winning coalitions can be thought of as equivalence classes: two sets that differ only on a set of $\lambda$-measure zero have the same status as winning or not winning. An implication is that the collection of winning coalitions cannot be defined without reference to the distribution of voter types. Furthermore, we incorporate a monotonicity condition into our definition: if $S \in \mathcal{W}$ and $T \in \Sigma$ satisfies $S \subseteq T$, then $T \in \mathcal{W}$. We say a coalition $S$ is blocking for $\mathcal{W}$ if its complement is not winning, and we let $\mathcal{B}$ denote the collection of blocking coalitions, i.e., $\mathcal{B}=\left\{S \in \Sigma: S^{c} \notin \mathcal{W}\right\}$. Note that $\mathcal{B}$ is itself a simple game, and that $\mathcal{W}$ and $\mathcal{B}$ are dual, in the sense that $\mathcal{W}$ consists of the coalitions blocking for $\mathcal{B}$.

The following properties of simple games will be used in the sequel. 
Definition 2 A simple game $\mathcal{W}$ satisfies the following conditions if it possesses the corresponding properties.

1. proper: $\mathcal{W} \subseteq \mathcal{B}$.

2. open from below: for all sequences $\left\{S_{n}\right\}$ in $\Sigma$ and all $S \in \mathcal{W}$, if $S_{n} \uparrow S$, then there exists $m$ such that, for all $n \geq m, S_{n} \in \mathcal{W}$.

3. closed from above: for all sequences $\left\{S_{n}\right\}$ in $\mathcal{W}$ and all $S \in \Sigma$, if $S_{n} \downarrow S$, then $S \in \mathcal{W}$.

4. liminf-open: for all sequences $\left\{S_{n}\right\}$ in $\Sigma$ with $\liminf S_{n} \in \mathcal{W}$, there exists $m$ such that, for all $n \geq m, S_{n} \in \mathcal{W}$.

5. limsup-closed: for all sequences $\left\{S_{n}\right\}$ in $\mathcal{W}$, $\lim \sup S_{n} \in \mathcal{W}$.

6. $\lambda$-open: for all $S \in \mathcal{W}$, there exists $\epsilon>0$ such that, for all $T \in \Sigma$, if $\lambda(S \backslash T) \leq \epsilon$, then $T \in \mathcal{W}$.

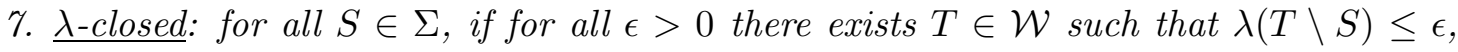
then $S \in \mathcal{W}$.

8. anonymous: for all $S \in \mathcal{W}$ and all $T \in \Sigma$, if $\lambda(T)=\lambda(S)$ then $T \in \mathcal{W}$.

9. semi-strong: for all $S \in \mathcal{B}$ and all $T \in \Sigma$, if $S \subseteq T$ and $\lambda(T \backslash S)>0$, then $T \in \mathcal{W}$.

10. strong: $\mathcal{B} \subseteq \mathcal{W}$.

Note that $\mathcal{W}$ is proper if and only if $S, T \in \mathcal{W}$ implies $\lambda(S \cap T)>0$. The next proposition establishes some connections between continuity properties of winning and blocking coalitions. Note that, by duality, the proposition implies that $\mathcal{W}$ is closed from above if and only if $\mathcal{B}$ is open from below, with similar dual results for liminf-open and limsup-closed simple games and for $\lambda$-open and $\lambda$-closed simple games.

\section{Proposition 7}

1. $\mathcal{W}$ is open from below if and only if $\mathcal{B}$ is closed from above.

2. $\mathcal{W}$ is liminf-open if and only if $\mathcal{B}$ is limsup-closed.

3. $\mathcal{W}$ is $\lambda$-open if and only if $\mathcal{B}$ is $\lambda$-closed.

Proof: To prove the first part of the proposition, note that $\mathcal{W}$ is open from below if and only if, for all sequences $\left\{S_{n}\right\}$ in $\Sigma$ and all $S \in \mathcal{W}, S_{n} \uparrow S$ implies there exists $m$ such that, for all $n \geq m, S_{n} \in \mathcal{W}$. Equivalently: if $S_{n} \uparrow S$ and there is some subsequence of $\left\{S_{n}\right\}$ (also indexed by $n$ ) such that, for all $n, S_{n} \notin \mathcal{W}$, then $S \notin \mathcal{W}$. Equivalently: if $S_{n}^{c} \downarrow S^{c}$ and there is some subsequence of $\left\{S_{n}^{c}\right\}$ such that, for all $n, S_{n}^{c} \in \mathcal{B}$, then $S^{c} \in \mathcal{B}$. And the latter means that $\mathcal{B}$ is closed from above. To prove the second part, note that $\mathcal{W}$ is 
liminf-open if and only if, for all sequences $\left\{S_{n}\right\}$ in $\Sigma$ with $\liminf S_{n} \in \mathcal{W}$, there exists $m$ such that, for all $n \geq m, S_{n} \in \mathcal{W}$. Equivalently: if there is some subsequence of $\left\{S_{n}\right\}$ (also indexed by $n$ ) such that, for all $n, S_{n} \notin \mathcal{W}$, then $\liminf S_{n} \notin \mathcal{W}$. Equivalently: if there is some subsequence of $\left\{S_{n}^{c}\right\}$ (also indexed by $n$ ) such that, for all $n, S_{n}^{c} \in \mathcal{B}$, then $\limsup S_{n}^{c}=\left(\liminf S_{n}\right)^{c} \in \mathcal{B}$. And the latter means that $\mathcal{B}$ is limsup-closed. To prove the third part, note that $\mathcal{W}$ is $\lambda$-open if and only if, for all $S \in \Sigma$, if for all $\epsilon>0$ there exists $T \notin \mathcal{W}$ such that $\lambda(S \backslash T) \leq \epsilon$, then $S \notin \mathcal{W}$. Equivalently: for all $S \in \Sigma$, if for all $\epsilon>0$ there exists $T \in \Sigma$ with $T^{c} \in \mathcal{B}$ such that $\lambda\left(T^{c} \backslash S^{c}\right) \leq \epsilon$, then $S^{c} \in \mathcal{B}$. And the latter means that $\mathcal{B}$ is $\lambda$-closed.

If $\Omega$ is finite, then every simple game is clearly $\lambda$-open, liminf-open, open from below, $\lambda$-closed, limsup-closed, and closed from above. The next proposition illustrates a general nesting of the first three of these concepts. By duality, of course, the next proposition immediately implies that, if $\mathcal{B}$ is $\lambda$-closed, then it is limsup-closed; and if $\mathcal{B}$ is limsup-closed, then it is closed from above.

\section{Proposition 8}

1. If $\mathcal{W}$ is $\lambda$-open, then it is liminf-open.

2. If $\mathcal{W}$ is liminf-open, then it is open from below.

Proof: Suppose $\mathcal{W}$ is $\lambda$-open, and take a sequence $\left\{S_{n}\right\}$ in $\Sigma$ such that $\lim \inf S_{n} \in \mathcal{W}$, i.e., $\bigcup_{n=1}^{\infty} \bigcap_{k=n}^{\infty} S_{n} \in \mathcal{W}$. Letting $T_{n}=\bigcap_{k=n}^{\infty} S_{n}$ and $T=\bigcup_{n=1}^{\infty} T_{n}$, we have $T_{n} \uparrow T$, implying $T \backslash T_{n} \downarrow \emptyset$. Therefore, $\lambda\left(T \backslash T_{n}\right) \rightarrow 0$. Since $\mathcal{W}$ is $\lambda$-open, there exists $m$ such that, for all $n \geq m, T_{n} \in \mathcal{W}$. Then, since $T_{n} \subseteq S_{n}$, we have $S_{n} \in \mathcal{W}$ for all $n \geq m$, as required. Now suppose $\mathcal{W}$ is liminf-open, and take a sequence $\left\{S_{n}\right\}$ in $\Sigma$ and $S \in \Sigma$ such that $S_{n} \uparrow S$. Clearly, $S=\liminf S_{n}$, so there exists $m$ such that, for all $n \geq m, S_{n} \in \mathcal{W}$, as required.

The next proposition establishes an implication of openness from below when voters are continuously distributed, namely, that we cannot have the collections of winning and blocking coalitions both open from below. Note the immediate implication, with Proposition

8 , that $\mathcal{W}$ and $\mathcal{B}$ cannot both be liminf-open or $\lambda$-open. By duality, these simple games cannot both be closed from above, limsup-closed, or $\lambda$-closed. The result also illustrates the restrictiveness of $\mathcal{W}$ being proper and strong: if both of those conditions hold, then $\mathcal{W}=\mathcal{B}$, so that $\mathcal{W}$ cannot be open from below. Though these conditions are relatively innocuous in finite electorates, in continuous electorates they are inconsistent with a basic continuity property of simple games.

Proposition 9 Assume $\lambda$ is non-atomic. If $\mathcal{W}$ is open from below, then $\mathcal{B}$ is not open from below.

Proof: Assume $\mathcal{W}$ is open from below, and suppose $\mathcal{B}$ is also open from below. Let $S_{1} \in \mathcal{W}$, and note that $\lambda\left(S_{1}\right)>0$, for otherwise $\emptyset \in \mathcal{W}$. Since $\lambda$ is non-atomic, for each natural 
number $n$, Lyapunov's Convexity Theorem (Aliprantis and Border (1999), Theorem 12.33) yields $S_{1}^{n} \in \Sigma$ such that $S_{1}^{n} \subseteq S_{1}$ and $\lambda\left(S_{1}^{n}\right)=1 / n$. Without loss of generality, we may assume $S_{1}^{n} \uparrow S_{1}$. Therefore, since $\mathcal{W}$ is open from below, the set

$$
E_{1}=\left\{\epsilon>0: \exists T \in \mathcal{W}: T \subseteq S_{1}, \lambda\left(S_{1} \backslash T\right) \geq \epsilon\right\}
$$

is nonempty. Let $\epsilon_{1}=\sup E_{1}>0$, and take $S_{2} \in \mathcal{W}$ such that $S_{2} \subseteq S_{1}$ and $\lambda\left(S_{1} \backslash S_{2}\right) \geq \epsilon_{1} / 2$. Since $\mathcal{W}$ is open from below and $\lambda$ is non-atomic,

$$
E_{2}=\left\{\epsilon>0: \exists T \in \mathcal{W}: T \subseteq S_{2}, \lambda\left(S_{2} \backslash T\right) \geq \epsilon\right\}
$$

is nonempty. Let $\epsilon_{2}=\sup E_{2}>0$, and take $S_{3} \in \mathcal{W}$ such that $S_{3} \subseteq S_{2}$ and $\lambda\left(S_{2} \backslash S_{3}\right) \geq \epsilon_{2} / 2$, and so on. Note that $\epsilon_{n} \rightarrow 0$. Define $S=\bigcap_{n=1}^{\infty} S_{n}$, so that $S_{n} \downarrow S$. If $S \notin \mathcal{W}$, then $S^{c} \in \mathcal{B}$. Because $\mathcal{B}$ is open from below and $S_{n}^{c} \uparrow S^{c}$, we then have $S_{n}^{c} \in \mathcal{B}$ for high enough $n$, contradicting $S_{n} \in \mathcal{W}$. Thus, $S \in \mathcal{W}$. Note that $\lambda(S)>0$, for otherwise $\emptyset \in \mathcal{W}$. By the above arguments, however, there exists $T \in \mathcal{W}$ such that $T \subseteq S$ and $\lambda(S \backslash T)>0$. Take $n$ high enough that $\epsilon_{n}<\lambda(S \backslash T)$, and note that $\lambda\left(S_{n} \backslash T\right) \geq \lambda(S \backslash T)>\epsilon_{n}$, a contradiction. Therefore, $\mathcal{B}$ is not open from below, as claimed.

If $\mathcal{W}$ is open from below and anonymous, then it is easy to see that it is simply defined by a quota $q \in[0,1]$, as follows:

$$
S \in \mathcal{W} \text { if and only if } \lambda(S)>q
$$

where $q \geq 1 / 2$ if $\mathcal{W}$ is proper. In fact, all quota rules of this form are actually $\lambda$-open. Majority rule, which is the special case with quota $q=1 / 2$, is always semi-strong: if $S \in \mathcal{B}$, then $\lambda(S) \geq 1 / 2$; then $S \subseteq T$ and $\lambda(T \backslash S)>0$ implies $\lambda(T)>1 / 2$, i.e., $T \in \mathcal{W}$. Of course, strong implies semi-strong. That the implication is strict can be seen in the important case of $\Omega$ finite: majority rule is always semi-strong but is not always strong, e.g., when $\lambda$ is the uniform measure and the number of voters is even. Note that $\mathcal{W}$ is strong if and only if $\mathcal{B}$ is proper; and $\mathcal{W}$ is proper if and only if $\mathcal{B}$ is strong. In models with a finite number $n$ of voters, majority rule with $n$ odd and dictatorship (i.e., $\mathcal{W}=\left\{S \in \Sigma: \omega^{\prime} \in S\right\}$ for some $\omega^{\prime} \in \Omega$ ) are examples of proper, strong simple games. If $\lambda$ is non-atomic, however, then majority rule is not strong and dictatorship is not proper. One way to see this is to note that both simple games are open from below, while majority rule is proper and dictatorship is strong, so the claim follows from Proposition 9. It is also straightforward to verify directly. In the case of majority rule, by Lyapunov's Convexity Theorem, there exists a coalition $S \in \Sigma$ such that $\lambda(S)=1 / 2$, so that $S, S^{c} \in \mathcal{B}$, so majority rule is not strong. Dictatorship is not even a well-defined simple game when $\left\{\omega^{\prime}\right\}$ has $\lambda$-measure zero, as the empty set would then also be winning, contrary to our definition. ${ }^{16}$

\footnotetext{
${ }^{16}$ For an example of an alternative to pure dictatorship, let $\Omega=[0,1]$, and define $\mathcal{W}$ to consist of the coalitions containing an open set around $\omega^{\prime}$. As long as $\lambda$ has a positive density, so that every open set has positive $\lambda$-measure, this simple game is proper. Moreover, given any continuous profile $\rho$, voter $\omega^{\prime}$ is, in fact, a dictator: if $a \pi\left(\omega^{\prime}\right) b$, then, by continuity, an open set around $\omega^{\prime}$ will share that strict preference, so $a$ is socially preferred to $b$. The collection of winning coalitions in this example is still not strong, however.
} 
Do proper, strong simple games exist when the electorate is a continuum and voters are continuously distributed? As the next proposition shows, the answer is affirmative. In fact, there exists a proper and strong simple game such that, for all $S, T \in \mathcal{W}$, we have $S \cap T \in \mathcal{W}$. The proof is a direct implication of Sikorski's (1964) Theorem 6.1 and surrounding discussion in his Section 6.

Proposition 10 There exists $\mathcal{W}$ that is proper and strong and such that, for all $S, T \in \mathcal{W}$, $S \cap T \in \mathcal{W}$.

Given the above model of an electorate, the next section analyzes the properties of social preferences generated by a simple game. Of note, we show that the proper and strong simple games established in the previous proposition are "rare" when the preferences of voters are sufficiently heterogeneous: such simple games are inconsistent with a fundamental continuity property of social preferences.

\section{Social Preferences}

Given an electorate and a simple game, social preferences over the set $A$ of alternatives are determined as follows. For $S \in \Sigma$, let

$$
P_{S}=\bigcap_{\omega \in S} \pi(\omega) \quad \text { and } \quad R_{S}=\bigcap_{\omega \in S} \rho(\omega),
$$

and define strict social preference, $P$, and weak social preference, $R$, as

$$
P=\bigcup_{S \in \mathcal{W}} P_{S} \quad \text { and } \quad R=\bigcup_{S \in \mathcal{B}} R_{S}
$$

That is, $a P b$ if and only if the set of voters who strictly prefer $a$ to $b$ is a winning coalition. Equivalently, $a R b$ if and only if the set of voters who weakly prefer $a$ to $b$ is blocking. Note that $\emptyset \notin \mathcal{W}$ implies that $P$ is irreflexive, and $\Omega \in \mathcal{B}$ implies $R$ is reflexive.

The next proposition gives weak conditions under which weak social preference $R$ is complete and strict social preference $P$ is the asymmetric part of $R$, i.e., $a P b$ if and only if $a R b$ and not $b R a$.

Proposition 11 Assume $A$ is Hausdorff and locally compact. If $\mathcal{W}$ is proper, then $R$ is complete and $P$ is the asymmetric part of $R$.

Proof: Take any $a, b \in A$, and suppose that neither $a R b$ nor $b R a$. Then, by definition, $S=\{\omega \in \Omega: a \rho(\omega) b\} \notin \mathcal{B}$ and $T=\{\omega \in \Omega: b \rho(\omega) a\} \notin \mathcal{B}$. Since $A$ is Hausdorff and locally compact, it follows from Aliprantis and Border's (1999) Theorem 2.63 that $\{R \in \mathcal{R}: a R b\}$ and $\{R \in \mathcal{R}: b R a\}$ are measurable. Since $\rho$ is measurable, it follows that $S$ and $T$ are measurable, and, therefore, we have $S^{c}, T^{c} \in \Sigma$. Then, by definition, we have $S^{c}, T^{c} \in \mathcal{W}$. But since each $\pi(\omega)$ is asymmetric, it follows that $S^{c}$ and $T^{c}$ are disjoint, contradicting the assumption that $\mathcal{W}$ is proper. Thus, $R$ is complete. That $P$ is the asymmetric part then follows by construction. 
Our main objective in this section is to give primitive conditions on the underlying electorate sufficient for the continuity properties of preferences used in Section 2, as well as for the "thin indifference" properties of Propositions 5 and 6. Our first result, on continuity of social preferences, is proved by McKelvey (1986) in his Lemma 2 for a finite number of voters: we extend the result to general electorates under our weakest continuity condition for simple games. ${ }^{17}$

Proposition 12 Assume $A$ is first countable. If $\mathcal{W}$ is open from below, then $P$ is open and $R$ is closed.

Proof: Suppose $a P b$, or equivalently, $(a, b) \in P$. Thus, there exists $S \in \mathcal{W}$ such that $(a, b) \in \bigcap_{\omega \in S} \pi(\omega)$. Let $\left\{G_{n}\right\}$ be a countable neighborhood base of $(a, b)$ in $A \times A$, and assume without loss of generality that it is decreasing. Since $\rho(\omega)$ is closed for each $\omega, \pi(\omega)$ is open for each $\omega$. Therefore, letting $S^{n}=\left\{\omega \in S: G_{n} \subseteq \pi(\omega)\right\}$, we have $S^{n} \uparrow S$. Since $\mathcal{W}$ is open from below, there exists $m$ such that, for all $n \geq m, S_{n} \in \mathcal{W}$. Therefore, $G_{n} \subseteq P$ for high enough $n$, so $P$ is open. That $R$ is closed then follows by definition.

From Proposition 7, if $\mathcal{B}$ is closed from above, then $P$ is open and $R$ is closed. If $A$ is a compact Hausdorff space, then $R(\cdot)$ is upper hemicontinuous as a correspondence. Though Proposition 12 yields openness of $P$, it cannot in general be simultaneously be applied to $\bigcup_{S \in \mathcal{B}} P_{S}$, the social preference generated by blocking coalitions: Proposition 9 has shown that, with a continuum of voters, $\mathcal{W}$ and $\mathcal{B}$ cannot both be open from below. We can, however, derive continuity properties of strict social preferences in simple games that are not open from below, if we impose topological conditions on the electorate and a condition restricting shared weak preferences across voters. Assuming $\Omega$ is a topological space, we denote by $S^{*}$ the support of $\lambda$, i.e., the smallest closed set with $\lambda$-measure one.

Definition 3 Limited shared weak preference (LSWP) holds if, for all $a, b \in A$ with $a \neq b$,

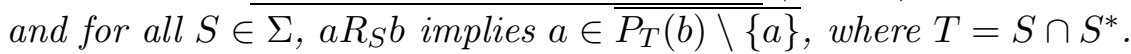

Thus, if every member of $S$ weakly prefers $a$ to $b \neq a$, then we can approximate $a$ by alternatives strictly preferred to $b$ by $\lambda$-almost every member of $S$. One condition sufficient for LSWP is evident. Assuming $A$ is a vector space, we say voter $\omega$ 's preferences are strictly convex if, for all $a \in A$, all $b \in \rho(\omega)(a) \backslash\{a\}$, and all $\alpha \in(0,1)$, we have $\alpha a+(1-\alpha) b \in \pi(\omega)(a)$. LSWP holds if $\lambda$ puts measure one on some closed subset of the voters with strictly convex preferences. Banks and Duggan $(1999,2000)$ give a number of examples of other environments satisfying LSWP in the finite-voter framework, including divide-the-dollar environments, private good economies, and mixed economies. As discussed in these papers, strict convexity does not hold in these environments, so that LSWP offers

\footnotetext{
${ }^{17}$ Shepsle and Weingast (1984) use McKelvey's result, but they consider "relative" majority rule ( $a$ is majority-preferred to $b$ if and only if more voters strictly prefer $a$ to $b$ than prefer $b$ to $a$ ), which does not possess this continuity property. Their results go through for "simple" majority rule, as defined in this paper.
} 
considerably more generality than the stronger condition. Note that LSWP implies that $A$ is infinite - in fact, it is inconsistent with the discrete topology.

The next result is stated for an arbitrary simple game $\mathcal{S}$. Under the assumptions of the lemma, Proposition 12 already shows that $P$ is open, giving us lower hemicontinuity of strict social preferences, but the lemma also applies when $\mathcal{S}=\mathcal{B}$.

Lemma 4 Assume $A$ is Hausdorff, second countable, and locally compact; $\Omega$ is a compact topological space and $\rho$ is continuous; and $L S W P$ holds. Let $\mathcal{S}$ be an arbitrary simple game. Then $\bigcup_{S \in \mathcal{S}} P_{S}(\cdot)$ is lower hemicontinuous as a correspondence.

Lower hemicontinuity of weak social preferences, critical for the nonemptiness of the undominated set, holds under the conditions of Lemma 4. Since those conditions are satisfied if the set of voters is finite with strictly convex preferences, the next result generalizes McKelvey's (1986) Lemma 4.

Proposition 13 Assume $A$ is Hausdorff, second countable, and locally compact; $\Omega$ is a compact topological space and $\rho$ is continuous; and LSWP holds. Then $P(\cdot)$ and $R(\cdot)$ are lower hemicontinuous as correspondences.

Proof: Lower hemicontinuity of $P(\cdot)$ follows directly from Lemma 4 by setting $\mathcal{S}=\mathcal{W}$. Now take any $a \in A$ and any $b \in R(a)$. If $b \neq a$, let $S \in \mathcal{B}$ be such that $b \in R_{S}(a)$. By LSWP, $b \in \overline{P_{T}(a) \backslash\{b\}}$, where $T=S \cap S^{*} \in \mathcal{B}$. Therefore, $b \in \overline{P_{T}(a)} \cup\{a\}$. Since $b \in R(a)$ was arbitrary, we have

$$
\{a\} \cup \bigcup_{S \in \mathcal{B}} P_{S}(a) \subseteq R(a) \subseteq\{a\} \cup \bigcup_{S \in \mathcal{B}} \overline{P_{S}(a)} \subseteq\{a\} \cup \overline{\bigcup_{S \in \mathcal{B}} P_{S}(a)} .
$$

The correspondence defined by $a \mapsto\{a\}$ is clearly lower hemicontinuous, as is $\bigcup_{S \in \mathcal{B}} P_{S}(\cdot)$, by Lemma 4. The union of these two correspondences is lower hemicontinuous, so $R(\cdot)$ differs from a lower hemicontinuous correspondence only at points of closure, implying that it is lower hemicontinuous.

The next result gives conditions under which upper sections of $R$ are compact. The assumptions on the set of alternatives are satisfied if $A$ is compact or if all voters have compact weak upper sections and $A$ is a subset of finite-dimensional Euclidean space. More precise sufficient conditions follow the proposition.

Proposition 14 Assume $A$ is first countable; for all $a \in A$ and all $\epsilon>0$, there exists a compact set $Y_{\epsilon} \subseteq A$ such that $\lambda\left(\left\{\omega \in \Omega: \rho(\omega)(a) \subseteq Y_{\epsilon}\right\}\right)>1-\epsilon$; and $\mathcal{W}$ is open from below. Then, for all $a \in A, R(a)$ is compact.

Proof: Let $\left\{Y_{1 / n}\right\}$ be a sequence of compact sets as in the assumption of the proposition, and without loss of generality assume the sequence is increasing. Take any $a \in A$, and 
suppose that, for each $n$, there exists $a_{n} \in R(a) \backslash Y_{1 / n}$. Let $S_{n}$ satisfy $S_{n} \in \mathcal{B}$ and $a_{n} R_{S_{n}} a$. Note that $S_{n} \subseteq T_{n}=\left\{\omega \in \Omega: \rho(\omega)(a) \nsubseteq Y_{1 / n}\right\}$, implying $T_{n} \in \mathcal{B}$, for all $n$. Note also that $\left\{T_{n}\right\}$ is a decreasing sequence and that $\lambda\left(T_{n}\right) \leq 1 / n$ for each $n$. Since $\mathcal{W}$ is open from below, it follows from Proposition 7 that $\mathcal{B}$ is closed from above, implying that $T=\bigcap_{n=1}^{\infty} T_{n} \in \mathcal{B}$. But $\lambda(T)=0$, implying $\emptyset \in \mathcal{B}$, which implies $\Omega \notin \mathcal{W}$, a contradiction. Therefore, $R(a) \subseteq Y_{1 / n}$ for some $n$. Since $R(a)$ is closed, by Proposition 12, it follows that $R(a)$ is compact.

The next proposition shows that compactness of voters' weak upper sections is essentially sufficient for the condition of Proposition 14, even in quite general spaces.

Proposition 15 Assume $A$ is Hausdorff, second countable, and locally compact; and, for all $a \in A, \lambda(\{\omega \in \Omega: \rho(\omega)(a)$ is compact $\})=1$. Then, for all $a \in A$ and all $\epsilon>0$, there exists a compact set $Y_{\epsilon} \subseteq A$ such that $\lambda\left(\left\{\omega \in \Omega: \rho(\omega)(a) \subseteq Y_{\epsilon}\right\}\right)>1-\epsilon$.

Proof: By Aliprantis and Border's (1999) Lemma 2.69, $A$ is $\sigma$-compact. By Aliprantis and Border's (1999) Corollary 2.70, $A$ is hemi-compact, i.e., there exist compact subsets $Y_{1}$, $Y_{2}, \ldots$, such that $A=\bigcup_{n=1}^{\infty} Y_{n}$, and for every compact $Y \subseteq A$, there exists $n$ such that $Y \subseteq \bigcup_{m=1}^{n} Y_{m}$. Now fix $a \in A$ and $\epsilon>0$. Let

$$
S_{n}=\left\{\omega \in \Omega: \rho(\omega)(a) \subseteq \bigcup_{m=1}^{n} Y_{m}\right\},
$$

and note that $\lambda\left(\bigcup_{n=1}^{\infty} S_{n}\right)=1$. Therefore, there exists $n$ such that $\lambda\left(S_{n}\right)>1-\epsilon$. Setting $Y_{\epsilon}=\bigcup_{m=1}^{n} Y_{m}$, the condition of the proposition is fulfilled.

We can strengthen the compactness results of Propositions 14 and 15 significantly, after strengthening our continuity assumption on the simple game: if $\mathcal{W}$ is liminf-open, then the image of every compact set, not just singletons, under the weak social preference correspondence $R(\cdot)$ is compact.

Proposition 16 Assume that $A$ is Hausdorff, second countable, and locally compact; for all $a \in A, \lambda(\{\omega \in \Omega: \rho(\omega)(a)$ is compact $\})=1$; and assume that $\mathcal{W}$ is liminf-open. Then, for all compact $Y \subseteq A$, the image $R(Y)=\bigcup_{a \in Y} R(a)$ is compact.

Proof: As in the proof of Proposition 15, there exist compact subsets $Y_{1}, Y_{2}, \ldots$, such that $A=\bigcup_{n=1}^{\infty} Y_{n}$, and for every compact $Y \subseteq A$, there exists $n$ such that $Y \subseteq \bigcup_{m=1}^{n} Y_{m}$. Without loss of generality, we assume $\left\{Y_{n}\right\}$ is increasing.

Now let $Y$ be a compact subset of $A$. Suppose that, for every $Y_{n} \subseteq A$, there exists $b_{n} \in R(Y) \backslash Y_{n}$. For each $n$, let $a_{n} \in Y$ satisfy $b_{n} \in R\left(a_{n}\right)$. Since $Y$ is compact, we may consider a convergent subsequence, still indexed by $n$, with limit $a \in Y$. For each $n$, let $S_{n}=\left\{\omega \in \Omega: b_{n} \rho(\omega) a_{n}\right\} \in \mathcal{B}$, and let $S=\bigcap_{n=1}^{\infty} \bigcup_{m=n}^{\infty} S_{m}$ be the limsup of this sequence. Since $\mathcal{W}$ is liminf-open, it follows from Proposition 7 that $\mathcal{B}$ is limsup-closed, so $S \in \mathcal{B}$. 
By assumption, $S^{\prime}=\left\{\omega \in \Omega: \rho(\omega)\left(a_{n}\right)\right.$ is compact for all $\left.n\right\}$ satisfies $\lambda\left(S^{\prime}\right)=1$, so that $T=S \cap S^{\prime} \in \mathcal{B}$. Suppose that $\lambda(T)>0$, and let $\epsilon=\lambda(T)$. From Proposition 8, it follows that $\mathcal{W}$ is open from below, and Proposition 15 then yields a compact set $Y_{\epsilon}$ such that $\lambda\left(\left\{\omega \in \Omega: \rho(\omega)(a) \subseteq Y_{\epsilon}\right\}\right)>1-\epsilon$. Take any $\omega \in T$, so that $\omega$ belongs to infinitely many $S_{n}$. Consider a subsequence, still indexed by $n$ for convenience, such that $\omega \in S_{n}$ for all $n$. Suppose $\rho(\omega)(a) \subseteq Y_{\epsilon}$. By Debreu's (1964) Proposition 3, there is a continuous utility representation $u: A \rightarrow \Re$ of $\rho(\omega)$. Choosing $n$ high enough that $Y_{\epsilon} \subseteq Y_{n}$, note that $b_{n} \notin Y_{n}$ implies $b_{n} \notin Y_{\epsilon}$, which implies $b_{n} \notin \rho(\omega)(a)$. Thus, $u(a)>u\left(b_{n}\right) \geq u\left(a_{n}\right)$ for sufficiently high $n$. By continuity, $u\left(a_{n}\right) \rightarrow u(a)$. Choosing any $n$ satisfying $u(a)>u\left(a_{n}\right)$, we therefore have $u\left(b_{n^{\prime}}\right) \geq u\left(a_{n}\right)$, i.e., $b_{n^{\prime}} \in \rho(\omega)\left(a_{n}\right)$, for infinitely many $n^{\prime}$. But $\rho(\omega)\left(a_{n}\right)$ is compact, and so $\rho(\omega)\left(a_{n}\right) \subseteq Y_{m}$ for some $m$. By construction, we must have $b_{n^{\prime}} \notin Y_{m}$ for all $n^{\prime} \geq m$, implying $b_{n^{\prime}} \notin \rho(\omega)\left(a_{n}\right)$ for all $n^{\prime} \geq m$, a contradiction. Therefore, we have $\rho(\omega)(a) \nsubseteq Y_{\epsilon}$ for all $\omega \in T$. But since $\lambda(T) \geq \epsilon$, we conclude that $\lambda\left(\left\{\omega \in \Omega: \rho(\omega)(a) \subseteq Y_{\epsilon}\right\}\right) \leq 1-\epsilon$, a contradiction. Therefore, $\lambda(T)=0$, and we conclude that $\emptyset \in \mathcal{B}$, which implies $\Omega \notin \mathcal{W}$, a contradiction.

Contrary to our initial supposition, there must exist $n$ such that $R(Y) \subseteq Y_{n}$. By Proposition 12, $R$ is closed, so the correspondence $R(\cdot): Y \rightarrow \rightarrow Y_{n}$, with domain restricted to $Y$ and range restricted to $Y_{n}$, is then a closed correspondence with compact Hausdorff range space. It is therefore upper hemicontinuous (Aliprantis and Border (1999), Theorem 16.12), and $R(Y)$, as the image of a compact set under an upper hemicontinuous correspondence, is compact (Aliprantis and Border (1999), Lemma 16.8).

We next turn to conditions under which social indifference curves are thin, i.e., $R(a)=$ $\overline{P(a)} \cup\{a\}$ for all $a$. An easily verified sufficient condition is that $\mathcal{W}$ is strong and all but a $\lambda$ measure zero set of voters have anti-symmetric weak preferences, meaning that $a \rho(\omega) b$ and $b \rho(\omega) a$ imply $a=b$. While anti-symmetry may be reasonable when the set of alternatives is finite, the assumption that no voter regards any two distinct alternatives as indifferent is quite restrictive when $A$ is infinite. Alternatively, McKelvey's (1986) Lemma 7 establishes that, if $\Omega$ is finite, if voters have strictly convex preferences over a subset of Euclidean space, and if $\mathcal{W}$ is strong, then thin social indifference will hold. While this result relies on more intuitive assumptions on voter preferences, it retains the assumption that $\mathcal{W}$ is strong, and we show below that this is extremely restrictive when there is a continuum of voters. Thus, we seek sufficient conditions for thin social indifference that relax that assumption to allow for such rules as majority voting. We use a lemma that gives conditions under which $P_{S}(a)$ is open for all compact coalitions $S$.

Lemma 5 Assume $A$ is Hausdorff, second countable, and locally compact; $\Omega$ is a topological space; and $\rho$ is continuous. Let $S \in \Sigma$ be compact. Then $P_{S}$ is open.

The next condition formalizes the notion that the preferences of voters are widely distributed. 
Definition 4 Dispersion holds if, for all distinct $a, b \in A$, for all $c \in A \backslash\{a, b\}$, and for every neighborhood $G$ of $c$, there exists $d \in G$ such that $\lambda(\{\omega \in \Omega: d \pi(\omega) a \pi(\omega) b\})>0$.

Dispersion is satisfied, for example, if voter preferences are Euclidean with ideal points distributed over Euclidean space by a strictly positive density. In that case, take any distinct $a, b \in A$, and take any other $c$. Given any neighborhood $G$ of $c$, we can find $d \in G$ such that $a, b, d$ are not collinear. Then there exists $e$ such that $\|e-d\|<\|e-a\|<\|e-b\|$, and these strict inequalities will hold for some open set around $e$. The set of voters with ideal points in this set has positive measure, fulfilling the condition. The next result shows that, under weak background conditions, dispersion of preferences is sufficient for thin social indifference curves, i.e., $R(a)=\overline{P(a)} \cup\{a\}$ for all $a \in A$. Thus, our condition acts much like the assumption that the number of voters is odd, in the finite framework. An implication is that, under reasonably weak conditions, Proposition 5 yields a simplification of the definition of covering in terms of social preferences: for $a$ to cover $b$, it is necessary and sufficient that both $a P b$ and $P(a) \subseteq P(b)$. Furthermore, for $a$ to dominate $b$, it is necessary and sufficient that $P(a)$ be a proper subset of $P(b)$.

Proposition 17 Assume that $A$ is Hausdorff, second countable, and locally compact; $\Omega$ is a compact topological space and $\rho$ is continuous; LSWP holds; dispersion holds; and $\mathcal{W}$ is proper, open from below, and semi-strong. Then $R(a)=\overline{P(a)} \cup\{a\}$ for all $a \in A$.

Proof: By Proposition 11, because $\mathcal{W}$ is proper, $P$ is the asymmetric part of $R$, so $P(a) \subseteq$ $R(a)$. Then, by Proposition 12, we have $\overline{P(a)} \cup\{a\} \subseteq R(a)$. Now take any $b \in R(a)$ with $b \neq a$, and let $S=\{\omega \in \Omega: b \rho(\omega) a\} \in \mathcal{B}$. Since $\rho$ is continuous, $S$ is closed, in fact compact. Take any open set $G$ around $b$. By LSWP, there is a sequence $\left\{c_{n}\right\}$ converging to $b$ such that $c_{n} \neq b$ for all $n$ and $c_{n} P_{T} a$, where $T=S \cap S^{*} \in \mathcal{B}$. Take any $c_{n} \in G$. Since $T$ is compact, Lemma 5 implies that $P_{T}(a)$ is open. Thus, $G^{\prime}=P_{T}(a) \cap G$ is an open set around $c_{n}$, so, by dispersion, there exists $d \in G^{\prime}$ such that $\lambda(\{\omega \in \Omega: d \pi(\omega) a \pi(\omega) b\})>0$. Let $T^{\prime}=T \cup\{\omega \in \Omega: d \pi(\omega) a \pi(\omega) b\}$. Since $\{\omega \in \Omega: d \pi(\omega) a \pi(\omega) b\} \cap T=\emptyset$, we have $\lambda\left(T^{\prime}\right)>\lambda(T)$, and our assumption that $\mathcal{W}$ is semi-strong implies $T^{\prime} \in \mathcal{W}$. Therefore, $d \in P(a)$. Since $G$ was arbitrary, we have $b \in \overline{P(a)}$.

Dispersion is also satisfied if $A$ is finite and if all linear orders of $A$ are present in the preferences of the electorate, in the sense that the set of voters with any given ordering has positive $\lambda$-measure. Because we use LSWP in the above proposition, however, we preclude the finite $A$ case. This is unavoidable, because we do not assume $\mathcal{W}$ is strong: if $A$ has the discrete topology, if $\mathcal{W}$ is majority rule, and if the number of voters is finite and even, then $R(a)=\overline{P(a)} \cup\{a\}(=P(a) \cup\{a\})$ will not hold generally, even if voter preferences are dispersed.

McKelvey's (1986) Lemma 7 on thin social indifference does indeed extend to the case of an infinite electorate, but it relies on the assumption that $\mathcal{W}$ is strong. Proposition 10 establishes the existence of a strong, proper simple game, so this extension of McKelvey's 
result is not vacuous, but its applicability is limited by Proposition 9, which shows that, with a continuum of voters, $\mathcal{W}$ proper and strong is inconsistent with $\mathcal{W}$ open from below. The latter is only a sufficient condition for continuous social preferences, so discontinuities are not necessarily implied. The following result shows, however, that when voters' preferences over a connected set of alternatives are sufficiently rich, all proper and strong simple games fail to generate continuous social preferences.

Proposition 18 Assume $A$ is Hausdorff, locally compact, and path-connected; for all $a, b \in$ $A, \lambda(\{\omega \in \Omega: \rho(\omega)(a)=\rho(\omega)(b)\})=0$; and there exists $a \in A$ with $P(a) \neq \emptyset$ and $P^{-1}(a) \neq \emptyset$. If $R$ is closed, then $\mathcal{W}$ is not both proper and strong.

Proof: Let $b P a$ and $a P c$. Let $f:[0,2] \rightarrow A$ be a continuous function satisfying $f(0)=a$, $f(1)=c$, and $f(2)=b$. Let $s=\sup \{x \in[0,2]: a P f(x)\}$. Since $b \in P(a)$, an open set, we have $1 \leq s<2$. By construction, there exists an increasing sequence $\left\{r_{n}\right\}$ in $[0,2]$ such that $r_{n} \rightarrow s$ and, for all $n, a P f\left(r_{n}\right)$. By Proposition 11 and the assumption that $\mathcal{W}$ is proper, we have $a R f\left(r_{n}\right)$ for all $n$. By continuity of $f, f\left(r_{n}\right) \rightarrow f(s)$, and, since $R$ is closed, $a R f(s)$. We may also take a decreasing sequence $\left\{t_{n}\right\}$ in $[0,2]$ such that $t_{n} \rightarrow s$ and, for all $n, f\left(t_{n}\right) R a$. Again, $f\left(t_{n}\right) \rightarrow f(s)$, and, since $R$ is closed, $f(s) R a$. So there exist $S, T \in \mathcal{B}$ such that $a R_{S} f(s)$ and $f(s) R_{T} a$. By assumption, $S^{\prime}=\{\omega \in S: a \pi(\omega) f(s)\}$ is $\lambda$-equivalent to $S$, so $S^{\prime} \in \mathcal{B}$, and similarly $T^{\prime}=\{\omega \in T: f(s) \pi(\omega) a\} \in \mathcal{B}$. If $\mathcal{W}$ is strong, then $\mathcal{B} \subseteq \mathcal{W}$, so $S^{\prime}, T^{\prime} \in \mathcal{W}$. But then $S^{\prime} \cap T^{\prime}=\emptyset$, so $\mathcal{W}$ is not proper.

Proposition 5 yields an alternative characterization of covering and dominance under the assumption of full social weak sections, i.e., $R^{-1}(a)=\overline{P^{-1}(a)} \cup\{a\}$. This condition is satisfied in McKelvey's (1986) model if voters have strictly convex preferences and $\mathcal{W}$ is strong, as long as $A$ is an open subset of $\Re^{n}$, a qualification omitted by McKelvey. To see that openness of $A$ is needed in the latter claim, consider the example of Figure 1, in which $A$ is a closed rectangle in $\Re^{2}$ with the relative topology, the electorate $\Omega=\{1,2,3\}$ consists of three voters, where $\lambda$ is uniform, voters have indifference curves as indicated (we draw "indifference curves" outside $A$ to clarify preferences involving $b$ ), and $\mathcal{W}$ is majority rule. Then $b$ is socially indifferent to $a$, in particular $b \in R^{-1}(a)$, but it is isolated from the alternatives to which $a$ is strictly socially preferred. Thus, $R^{-1}(a) \neq \overline{P^{-1}(a)} \cup\{a\}$. To see the consequences for the definition of covering, note that $R(c) \subseteq R(a)$, yet $b \in$ $P(c) \backslash P(a)$. Therefore, $R(c) \subseteq R(a)$ does not imply $P(c) \subseteq P(a)$, contrary to McKelvey's (1986) Proposition 3.3, demonstrating that the condition used in part 2 of Proposition 5 is needed for the result.

For an even simpler example, where voter preferences are convex but not strictly so, consider the divide-the-dollar model with a finite set of voters: assume $\Omega=\left\{\omega_{1}, \ldots, \omega_{n}\right\}$ consists of an odd number $n \geq 3$ voters, where $\lambda$ is uniform; the set of alternatives is $A=\left\{a \in \Re_{+}^{n}: \sum_{i=1}^{n} a_{i}=1\right\}$, the unit simplex in $\Re^{n}$; for each voter $\omega_{i}, a_{i} \pi\left(\omega_{i}\right) b_{i}$ if and only if $a_{i}>b_{i}$; and $\mathcal{W}$ is majority rule. Then, letting $a=\left(0, \frac{2}{n-1}, 0, \ldots, \frac{2}{n-1}, 0\right)$, we have $R(a)=A$. Note that $b=\left(0, \frac{1}{n-1}, \ldots, \frac{1}{n-1}\right) \in R^{-1}(a)$, but $b$ is isolated from the alternatives 


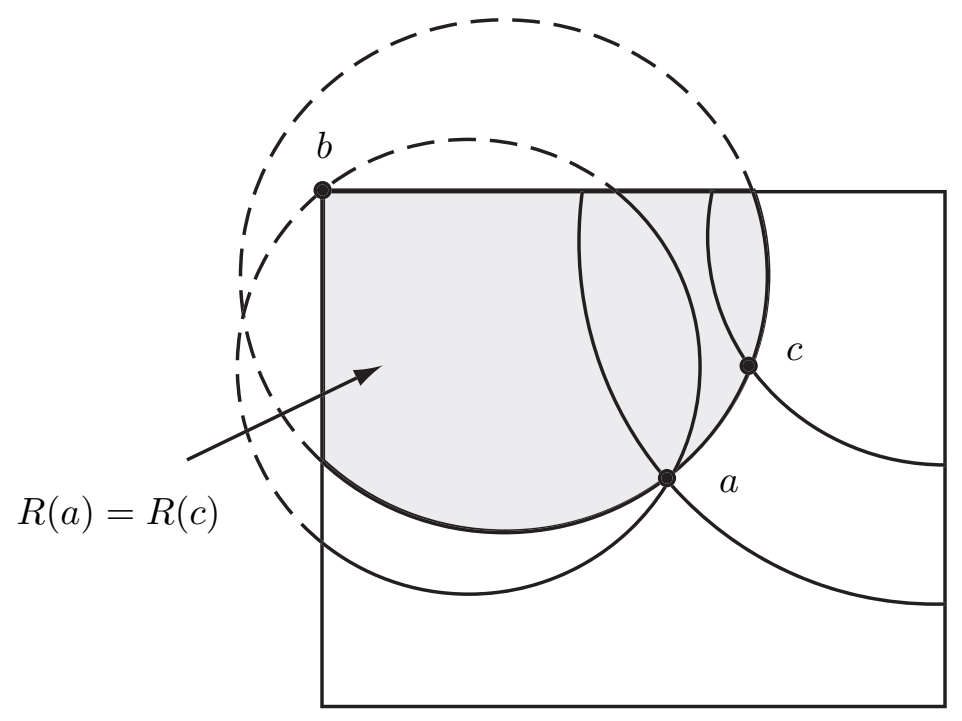

Figure 1: Violation of McKelvey's implicit assumption

to which $a$ is socially preferred - in fact, $P^{-1}(a)=\emptyset$. Thus, $R^{-1}(a) \neq \overline{P^{-1}(a)} \cup\{a\}$. As a consequence, letting $c=(1,0, \ldots, 0)$, we have $R(c) \subseteq R(a)$ but $b \in P(c) \backslash P(a)$. Therefore, $R(c) \subseteq R(a)$ does not imply $P(c) \subseteq P(a)$, creating the possibility of multiple uncovered sets, noted by Penn (2002) in this model.

As Propositions 9 and 18 show, the assumption that $\mathcal{W}$ is strong is quite restrictive when there is a continuum of voters. Thus, its usefulness in obtaining the condition $R^{-1}(a)=\overline{P^{-1}(a)} \cup\{a\}$ is limited in our framework. The next result verifies that the assumption that $\mathcal{W}$ is strong can be replaced by dispersion of preferences and the much weaker assumption that $\mathcal{W}$ is semi-strong. Recall that the latter condition is satisfied, for example, by majority rule. Under the conditions of Proposition 17 and the following result, we obtain the alternative characterizations of covering in Proposition 5 and the two-step principles of Proposition 6.

Proposition 19 Assume $A$ is an open, convex subset of $\Re^{n}$ with the relative topology; $\Omega$ is a compact topological space and $\rho$ is continuous; $S^{*} \subseteq\{\omega \in \Omega: \rho(\omega)$ is strictly convex $\}$; dispersion holds; and $\mathcal{W}$ is proper, open from below, and semi-strong. Then $R^{-1}(a)=$ $\overline{P^{-1}(a)} \cup\{a\}$ for all $a \in A$.

Proof: By Proposition 11, because $\mathcal{W}$ is proper, it follows that $P$ is the asymmetric part of $R$, so $P^{-1}(a) \subseteq R^{-1}(a)$. Then, by Proposition 12 , we have $\overline{P^{-1}(a)} \cup\{a\} \subseteq R^{-1}(a)$. Now take any $b \in R^{-1}(a)$ with $b \neq a$, so $S=\{\omega \in \Omega: a \rho(\omega) b\} \in \mathcal{B}$. Take any open set $G$ around $b$, and assume without loss of generality that $G$ is convex. Since $\rho$ is continuous, $S$ is closed, in fact compact. Therefore, $T=S \cap S^{*} \in \mathcal{B}$ is also compact, and Lemma 5 implies that $P_{T}^{-1}(a)$ is open. Since $A$ is open as a subset of $\Re^{n}$, there exists a non-empty, convex, open set $G^{\prime} \subseteq \Re^{n}$ such that $b \in G^{\prime} \subseteq A$. Let $c \in G \cap G^{\prime}$ satisfy $c=(1-\alpha) a+\alpha b$ for some $\alpha>1$. Let 
$d=(1 / 2) b+(1 / 2) c$, which is an element of $G \cap G^{\prime}$. By strict convexity of voter preferences, therefore, we have $a \pi(\omega) d \pi(\omega) c$ for all $\omega \in T$. In particular, $c, d \in P_{T}^{-1}(a)$. Since $a, c$, and $d$ are distinct, dispersion yields $e \in G \cap G^{\prime} \cap P_{T}^{-1}(a)$ such that $\lambda(\{\omega \in \Omega: e \pi(\omega) a \pi(\omega) c\})>0$. Let $T^{\prime}=T \cup\{\omega \in \Omega: e \pi(\omega) b \pi(\omega) c\}$. Since $\{\omega \in \Omega: e \pi(\omega) a \pi(\omega) c\} \cap T=\emptyset$, we have $\lambda\left(T^{\prime}\right)>\lambda(T)$, and our assumption that $\mathcal{W}$ is semi-strong implies $T^{\prime} \in \mathcal{W}$. Therefore, $c \in P^{-1}(a) \cap G$. Since $G$ was arbitrary, we have $b \in \overline{P^{-1}(a)}$.

We return to the issue of the two-step principle in the next section, where we tie together our earlier results and examine the properties of the core, undominated set, and uncovered set as the preferences of voters vary.

\section{Electoral Competition}

Given a policy space $A$ and an electorate $\Omega$ with winning coalitions $\mathcal{W}$, consider a competition between two office-motivated parties, where each party strategically chooses its platform in the set $A$ in order to maximize its chances of winning the election. Precisely, the two parties play a symmetric, zero-sum game, in which $A$ is the common set of pure strategies and the payoff of, say, party 1 from the strategy profile $(a, b)$ is defined by

$$
u(a, b)=\left\{\begin{array}{cl}
1 & \text { if } a P b \\
-1 & \text { if } b P a \\
0 & \text { else }
\end{array}\right.
$$

where here $a$ denotes the platform of party 1 and $b$ the platform of party 2 . It is easy to see that $a^{*}$ is an optimal play in this game if and only if $a^{*}$ is not defeated by a winning coalition, i.e., $a^{*} \in K$. This means that the existence of a Nash equilibrium in pure strategies is equivalent to the nonemptiness of the core. On the other hand, the set of strategies obtained after deletion of the weakly dominated strategies is precisely the undominated set. The uncovered set, which is a superset (sometimes proper) of the undominated set, does not have a direct game-theoretic interpretation. But as demonstrated by Banks, Duggan, and Le Breton (2000) for a class of games including the two-party competition game described above, the support of every Nash equilibrium in mixed strategies is contained in the uncovered set.

In this section, we analyze properties of these choice sets as electoral preferences vary. Fixing the winning coalitions $\mathcal{W}$, we write $P[\rho]$ and $R[\rho]$ for the strict and weak social preferences determined by profile $\rho$. We write $K[\rho], U C[\rho]$, and $U D[\rho]$ for the core, uncovered set, and undominated set of the social preference relations $P[\rho]$ and $R[\rho]$. We extend previous results on generic emptiness of the core to the general spatial model, and we establish nonemptiness of the uncovered and undominated sets in the general model. We then show that the three above correspondences are upper hemicontinuous at profiles with a non-empty and externally stable core. Thus, though small perturbations of preferences may (and usually will) lead to an empty core, this result, with our above observations on electoral 
competition, suggests that electoral outcomes will change continuously. Finally, we provide conditions under which the undominated set correspondence is lower hemicontinuous.

The next lemma on continuity of social preferences is essential to the analysis. Assume that $\Omega$ is a Polish space, i.e., a complete and separable metric space, and that $\sigma$ is the corresponding Borel $\sigma$-algebra. Let $d$ be the metric on $\Omega$. Assuming $A$ is a locally compact Polish space, the closed convergence topology on $\mathcal{R}$ is metrizable (Aliprantis and Border (1999), Corollary 3.81), and we let $\tilde{d}$ denote a metric that generates that topology. Let $\mathbf{P}$ be the space of preference profiles, and define the semi-metric $\Delta$ on $\mathbf{P}$ as follows:

$$
\Delta\left(\rho, \rho^{\prime}\right)=\int_{\Omega} \tilde{d}\left(\rho(\omega), \rho^{\prime}(\omega)\right) \lambda(d \omega)
$$

for $\rho, \rho^{\prime} \in \mathbf{P}$. We will freely identify profiles that differ on a $\lambda$-measure zero set of voters, in which case we may view $\Delta$ as a metric. We then say a sequence $\left\{\rho_{n}\right\}$ of profiles converges to profile $\rho$, written $\rho_{n} \rightarrow \rho$, if $\Delta\left(\rho_{n}, \rho\right) \rightarrow 0$. Under the strongest of our continuity conditions on simple games, we obtain the following continuity result for social preferences.

Lemma 6 Assume $A$ is a locally compact, complete, separable metric space; $\Omega$ is a complete and separable metric space; and $\mathcal{W}$ is $\lambda$-open. Let $\rho_{n} \rightarrow \rho$, let $a_{n} \rightarrow a$, and let $b_{n} \rightarrow b$. If $a_{n} R\left[\rho_{n}\right] b_{n}$ for all $n$, then $a R[\rho] b$.

When there is a finite number of voters, different formulations of the assertion that the core is generically empty have been provided. Some authors, in the vein of Plott's (1967) seminal contribution, assume that voter preferences are differentiable and perhaps convex. Their results provide characterizations of core points in terms of differentiability properties, which are evidently quite difficult to satisfy when the dimensionality of the space of alternatives is sufficiently high (at least two, for the case of majority rule with an odd number of voters). Other authors do not assume differentiability or convexity of voter preferences and prove the generic emptiness of the core directly while imposing only continuity on voter preferences (Rubinstein (1979), Cox (1984), Le Breton (1987)). In the latter work, because the space of voter preferences is richer, no dimensionality restrictions are needed. McKelvey, Ordeshook, and Ungar (1980) have proved that Plott's characterization in terms of symmetry of voter gradients at core points holds true while allowing for a measure space of voters. Our next result plays the complementary role for the above-cited literature on generic emptiness of the core when voter preferences are restricted only by continuity. The implication for electoral competition is that pure strategy equilibria of the electoral game will almost never exist.

When $\Omega$ is finite, we say $\mathcal{W}$ is non-collegial if $\cap \mathcal{W}=\emptyset$. When there is a continuum of massless voters, however, every simple game is non-collegial according to this definition: for each $\omega \in \Omega, \Omega \backslash\{\omega\} \in \mathcal{W}$, so $\mathcal{W}$ has empty intersection. We extend the usual definition as follows. We say $\mathcal{W}$ is non-collegial if, for every $S \in \Sigma$, there exists a finite measurable partition, $\left\{S_{1}, \ldots, S_{M}\right\}$, of $S$ such that, for all $m, \Omega \backslash S_{m} \in \mathcal{W}$. Given a non-collegial simple game, note that, if $S \in \Sigma$ is an atom, then $S \notin \mathcal{B}$. On the other hand, if $\lambda$ is 
atomless, then, by Aliprantis and Border's (1999) Theorem 12.34, for all $\epsilon>0$, there exists a finite measurable partition, $\left\{S_{1}, \ldots, S_{M}\right\}$, of $\Omega$ such that $\lambda\left(S_{m}\right)<\epsilon$ for all $m$. Thus, if $\lambda$ is atomless and there exists $\epsilon>0$ such that $\lambda(S)>1-\epsilon$ implies $S \in \mathcal{W}$, then $\mathcal{W}$ is non-collegial.

Proposition 20 Assume $A$ is a compact and convex subset of some Euclidean space with $|A|>2 ; \Omega$ is a complete and separable metric space; and $\mathcal{W}$ is proper, non-collegial, and $\lambda$-open. Then the set

$$
\mathbf{K}=\{\rho \in \mathbf{P}: K[\rho] \neq \emptyset\}
$$

is closed and nowhere dense in $\mathbf{P}$ in the $\Delta$ metric.

Proof: We first prove $\mathbf{K}$ is closed. Let $\rho_{n} \rightarrow \rho$ with $\rho_{n} \in \mathbf{K}$ for all $n$. Let $a_{n} \in K\left[\rho_{n}\right]$ for each $n$. Since $A$ is compact, there is a subsequence $\left\{a_{n_{k}}\right\}$ converging to some limit $a$. Take any $b \in A$. Since $R\left[\rho_{n_{k}}\right]$ is complete, by Proposition 11, we have $a_{n_{k}} R\left[\rho_{n_{k}}\right] b$ for all $k$. Then Lemma 6 implies $a R[\rho] b$. Since $b$ is arbitrary here, we have $a \in K[\rho] \neq \emptyset$.

To prove that $\mathbf{K}$ has empty interior, take $\rho \in \mathbf{K}$ and $\epsilon>0$. We will show that there exists $\rho_{\epsilon}$ such that $\Delta\left(\rho_{\epsilon}, \rho\right) \leq \epsilon$ and $\rho_{\epsilon} \notin \mathbf{K}$. Let $c=\sup \left\{\tilde{d}\left(R, R^{\prime}\right): R, R^{\prime} \in \mathcal{R}\right\} \cup\{1\}$, which is finite, since $A$ is compact. From Lusin's Theorem (Aliprantis and Border (1999), Theorem 10.8), there exists a compact subset $Y_{\epsilon}$ of $\Omega$ such that $\lambda\left(Y_{\epsilon}\right) \geq 1-(\epsilon / 4 c)$ and $\rho$, restricted to $Y_{\epsilon}$, is continuous. Since $Y_{\epsilon}$ is compact, $\rho$ is uniformly continuous on $Y_{\epsilon}$. Let $\delta>0$ be such that $d\left(\omega, \omega^{\prime}\right) \leq \delta$ implies $\tilde{d}\left(\rho(\omega), \rho\left(\omega^{\prime}\right)\right) \leq \epsilon / 4$. Letting $B_{\delta}(\omega)$ denote the open $d$-ball with radius $\delta$ centered at $\omega,\left\{B_{\delta}(\omega): \omega \in Y_{\epsilon}\right\}$ is an open cover of $Y_{\epsilon}$. By compactness, it has a finite subcover, say $\left\{B_{1}, \ldots, B_{M}\right\}$. Let $S_{1}=B_{1}$, let

$$
S_{m}=B_{m} \backslash \bigcup_{j=1}^{m-1} B_{j}
$$

for $m=2, \ldots, M$, and let $S_{M+1}=\Omega \backslash Y_{\epsilon}$. The family $\left\{S_{m}\right\}$ is a measurable partition of $\Omega$. Since $\mathcal{W}$ is non-collegial, for each $m$ there is a finite partition $\left\{S_{m}^{j}: j=1, \ldots, J_{m}\right\}$ such that, for all $j, S_{m}^{j} \notin \mathcal{B}$. Now let $\mathcal{T}$ be the finite partition

$$
\mathcal{T}=\left\{S_{m}^{j}: j=1, \ldots, J_{m}, m=1, \ldots, M+1\right\},
$$

and index the elements of $\mathcal{T}$ as $T_{i}, i=1, \ldots, n$. For each $i$, let $R_{i}^{\prime}$ be an arbitrary element of $\left\{\rho(\omega): \omega \in T_{i}\right\}$, and define the profile $\rho_{\epsilon}^{\prime}$ as $\rho_{\epsilon}^{\prime}(\omega)=R_{i}^{\prime}$ for all $\omega \in T_{i}$. Since

$$
\begin{aligned}
\Delta\left(\rho, \rho_{\epsilon}^{\prime}\right) & =\int_{\Omega \backslash Y_{\epsilon}} \tilde{d}\left(\rho(\omega), \rho_{\epsilon}^{\prime}(\omega)\right) \lambda(d \omega)+\sum_{i=1}^{n} \int_{T_{i} \cap Y_{\epsilon}} \tilde{d}\left(\rho(\omega), \rho_{\epsilon}^{\prime}(\omega)\right) \lambda(d \omega) \\
& \leq \lambda\left(\Omega \backslash Y_{\epsilon}\right) c+\lambda\left(Y_{\epsilon}\right) \epsilon / 4
\end{aligned}
$$

it follows that $\Delta\left(\rho, \rho_{\epsilon}^{\prime}\right) \leq \epsilon / 2$. 
Now define a finite simple game $\mathcal{W}^{n}$ on the set $\{1, \ldots, n\}$ as follows: for $S \subseteq\{1, \ldots, n\}$, let

$$
S \in \mathcal{W}^{n} \text { if and only if } \bigcup_{i \in S} T_{i} \in \mathcal{W} .
$$

Thus, by choice of $\left\{T_{i}\right\}, \mathcal{W}^{n}$ is non-collegial. Given any finite profile $\left(R_{1}, \ldots, R_{n}\right)$, let

$$
P^{n}\left[R_{1}, \ldots, R_{n}\right]=\bigcup_{S \in \mathcal{W}^{n}} \bigcap_{i \in S} P_{i},
$$

and define the core for $\mathcal{W}^{n}$ as $K^{n}\left[R_{1}, \ldots, R_{n}\right]=\left\{a \in A: P^{n}\left[R_{1}, \ldots, R_{n}\right](a)=0\right\}$. Since $\mathcal{W}^{n}$ is non-collegial, it follows from Le Breton (1987) that there exists a finite profile $\left(R_{1}, \ldots, R_{n}\right)$ such that $K^{n}\left[R_{1}, \ldots, R_{n}\right]=\emptyset$ and $\tilde{d}\left(R_{i}, R_{i}^{\prime}\right) \leq \epsilon / 2$ for all $i=1, \ldots, n$. Define the profile $\rho_{\epsilon}: \Omega \rightarrow \mathcal{R}$ by $\rho_{\epsilon}(\omega)=R_{i}$ for all $\omega \in T_{i}$ and all $i=1, \ldots, n$. Clearly, $K\left[\rho_{\epsilon}\right]=K^{n}\left[R_{1}, \ldots, R_{n}\right]=\emptyset$, so we have $\rho_{\epsilon} \notin \mathbf{K}$ and $\Delta\left(\rho, \rho_{\epsilon}\right) \leq \epsilon$, as required.

This negative result for pure strategy equilibria leads to interest in alternative solutions for the electoral game: if parties eliminate weakly dominated strategies, for example, then they will choose platforms in the undominated set; and if parties play mixed strategy equilibria, then they will choose uncovered platforms. Thus, these choice sets may yield useful bounds on electoral outcomes. The next results show that, in contrast to the core, the uncovered and undominated sets are non-empty quite generally.

Proposition 21 Assume $A$ is first countable; for all $a \in A$ and all $\epsilon>0$, there exists a compact set $Y_{\epsilon}$ such that $\lambda\left(\left\{\omega \in \Omega: \rho(\omega)(a) \subseteq Y_{\epsilon}\right\}\right)>1-\epsilon$; and $\mathcal{W}$ is open from below. Then $U C[\rho] \neq \emptyset$.

Proof: By Proposition 14, each $R[\rho](a)$ is compact. By Proposition 2, therefore, we have $U C[\rho]=U C(R[\rho]) \neq \emptyset$.

Nonemptiness of the undominated set and external stability of these sets follow if we impose topological conditions on the electorate. We write $D[\rho]$ and $C[\rho]$ for the dominance and covering relations determined by $R[\rho]$ and $P[\rho]$.

Proposition 22 Assume $A$ is Hausdorff, second countable, and locally compact; $\Omega$ is a compact topological space and $\rho$ is continuous; LSWP holds at $\rho$; for all $a \in A$ and all $\epsilon>0$, there exists a compact set $Y_{\epsilon}$ such that $\lambda\left(\left\{\omega \in \Omega: \rho(\omega)(a) \subseteq Y_{\epsilon}\right\}\right)>1-\epsilon$; and $\mathcal{W}$ is open from below.

1. $U D[\rho] \neq \emptyset$.

2. If $a \notin U D[\rho]$, then there exists $b \in U D[\rho]$ such that $b D[\rho] a$.

3. If $a \notin U C[\rho]$, then there exists $b \in U C[\rho]$ such that $b C[\rho] a$. 
Proof: By Proposition 14, each $R[\rho](a)$ is compact and therefore closed. By Proposition $13, R[\rho](\cdot)$ is lower hemicontinuous as a correspondence, and then Proposition 3 yields $U D[\rho] \neq \emptyset$. External stability of $U D[\rho]$ and $U C[\rho]$ then follows from Proposition 4.

Using Propositions 5, 17, and 19, it is straightforward to provide assumptions on primitives of the social choice model that yield equivalent formulations of covering and dominance. We omit those applications of our results and proceed to the two-step principle, the subject of Proposition 6. The next proposition ties together our results on this issue. In parts 2 and 3 , we are able to relax openness of $A$, from Proposition 19, by focusing on undominated or uncovered alternatives satisfying an interiority requirement: we require that all alternatives weakly socially preferred to a given $a$ lie in the interior of $A$.

Proposition 23 Assume $A$ is Hausdorff and locally compact; and $\mathcal{W}$ is proper.

$$
\text { 1. } \bigcap_{a \in A}\left(P[\rho]^{2}(a) \cup\{a\}\right) \subseteq U D[\rho] \subseteq U C[\rho] \subseteq \bigcap_{a \in A} R[\rho]^{2}(a)
$$

In addition, assume $A$ is a convex subset of $\Re^{n}$ with the relative topology; $\Omega$ is a compact topological space and $\rho$ is continuous; $S^{*} \subseteq\{\omega \in \Omega: \rho(\omega)$ is strictly convex $\}$; dispersion holds; and $\mathcal{W}$ is open from below and semi-strong. Let $a \in A$ be such that $R[\rho](a)$ is contained in the interior of $A$ in $\Re^{n}$.

2. If $a \in U D[\rho]$ and $b \notin U D[\rho]$, then $a \in P[\rho]^{2}(b)$. If $a \in U C[\rho]$ and $b \notin U C[\rho]$, then $a \in P[\rho]^{2}(b)$.

Moreover, assume that, for all $a, b \in A, P[\rho](a)=P[\rho](b)$ implies $a=b$.

3. If $a \in U D[\rho]$ and $b \in A \backslash\{a\}$, then $a \in P[\rho]^{2}(b)$.

Proof: The first part follows directly from Propositions 6 and 11. For the second part, let $a$ be as in the proposition. Since $R[\rho](a)$ is nonempty, it follows that $A$ has nonempty interior in $\Re^{n}$, which we denote $\hat{A}$. Since $A$ is convex, this implies that $A$ is contained in the closure of $\hat{A}$. Suppose $a \in U D[\rho]$ and $b \notin U D[\rho]$. Let $\hat{\rho}(\omega)$ be the restriction of $\rho(\omega)$ to $\hat{A}$; let $\hat{P}$ and $\hat{R}$ be the social preferences induced by the profile $\hat{\rho}$; define the dominance relation $\hat{D}$ with respect to these social preferences; and let $\widehat{U D}$ denote the corresponding undominated set. Note that $\hat{\rho}$ is continuous, satisfies LSWP, and satisfies dispersion. To see that $a \in \widehat{U D}$, suppose $c \hat{D} a$ for some $c \in \hat{A}$, so $\hat{P}(c) \subseteq \hat{P}(a)$ and $\hat{R}(c) \subseteq \hat{R}(a)$, at least one inclusion strict. Take any $d \in P[\rho](c)$. If $a P[\rho] d$, then $d \in P[\rho]^{-1}(a) \cap P[\rho](c)$, an open set in the relative topology on $A$, by Proposition 12. Then there exists some $e \in$ $P[\rho]^{-1}(a) \cap P[\rho](c) \cap \hat{A}$, which implies $a \hat{P} e \hat{P} c$. But then $c \hat{D} a$ implies $e \hat{P} a$, a contradiction. Thus, $d R[\rho] a$. Then, by assumption, we have $d \in \hat{A}$, and from $d P[\rho] c$ we have $d \hat{P} c$. With $c \hat{D} a$, this implies $d \hat{P} a$, which implies $d P[\rho] a$. Since $d$ was an arbitrary element of $P[\rho](c)$, we see that $P[\rho](c) \subseteq P[\rho](a)$. Take any $d \in R[\rho](c)$. If $a P[\rho] d$, then, by Proposition 17 , either $a P[\rho] c$ or $P[\rho]^{-1}(a) \cap \overline{P[\rho](c)} \neq \emptyset$. In the former case, we then have $a \hat{P} c$, which contradicts 
$c \hat{D} a$. In the latter case, there exists some $e \in P[\rho]^{-1}(a) \cap P[\rho](c) \cap \hat{A}$. Then, by the above argument, we again find $d R[\rho] a$, and we see that $R[\rho](c) \subseteq R[\rho](a)$. Moreover, at least one of these inclusions is strict. Therefore, $c D a$, contradicting $a \in U D$. We conclude that $a \in \widehat{U D}$, as claimed. If $b \in A \backslash \hat{A}$, then we have $a P[\rho] b$, by assumption. Otherwise, $b \in \hat{A}$, and then Propositions 6, 17, and 19 yield $c \in \hat{A}$ such that $a \hat{P} c \hat{P} b$, implying $a P[\rho] c P[\rho] b$. If $a \in U C[\rho]$, then a similar argument establishes $a \in P[\rho]^{2}(b)$. For the third part of the proposition, suppose $a \in U D[\rho]$, and let $b \neq a$. If $b \notin U D[\rho]$, the result follows from

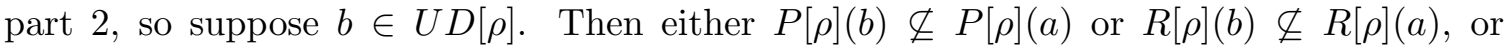
$P[\rho](a)=P[\rho](b)$. The first two cases proceed as in part 2 , while the last case is ruled out by assumption.

Of interest, then, is the approximate location of these sets. Miller (1980) and Shepsle and Weingast (1984) have shown, for the case of tournaments and the spatial model in Euclidean space, that the uncovered set is contained in the set of Pareto optimal alternatives. The result follows from the same logic in the general model. Here, given an electorate with preference profile $\rho$, we define the Pareto dominance relation, denoted $\tilde{P}[\rho]$, as follows: $a \tilde{P}[\rho] b$ if and only if $\lambda(\{\omega \in \Omega: a \pi(\omega) b\})=1$, i.e., almost all voters strictly prefer $a$ to $b$. The Pareto optimal alternatives consist of the $\tilde{P}[\rho]$-maximal elements:

$$
P O[\rho]=\{a \in A: \tilde{P}[\rho](a)=\emptyset\} .
$$

The next result establishes, in the framework of general electorates, that the uncovered set, and therefore the undominated set, is contained in the Pareto optimals.

Proposition 24 Assume $\mathcal{W} \neq \emptyset$. Then $U C[\rho] \subseteq P O[\rho]$.

Proof: Take any $a \in U C[\rho]$, and suppose $b \tilde{P}[\rho] a$ for some $b \in A$. We claim that $b C[\rho] a$. First, note that there exists $S \in \mathcal{W}$, and that $T=\{\omega \in S: b \pi(\omega) a\}$ satisfies $\lambda(T)=\lambda(S)$. Therefore, $T \in \mathcal{W}$, and $b P[\rho] a$. Second, take any $c \in R(b)$, and consider $S \in \mathcal{B}$ such that $c R_{S} b$. Defining $T=\{\omega \in S: b \pi(\omega) a\}$, we have $\lambda(T)=\lambda(S)$, so $T \in \mathcal{B}$. Moreover, since each $\rho(\omega)$ is a weak order, we have $c P_{S} a$, which implies $c \in R(a)$. Therefore, $R(b) \subseteq R(a)$. Finally, take any $c \in P(b)$, and consider $S \in \mathcal{W}$ such that $c P_{S} b$. Then the argument of the previous case yields $T \in \mathcal{W}$ such that $c P_{S} a$, i.e., $c \in P(a)$. Therefore, $P(b) \subseteq P(a)$, and we conclude that $b C[\rho] a$, a contradiction. Therefore, we have $a \in P O[\rho]$, as claimed.

We now examine the continuity properties of the uncovered set correspondence. The next result demonstrates that, at profiles $\rho$ such that $K[\rho]$ is nonempty and is strongly externally stable, the correspondence $U C[\cdot]$ is upper hemicontinuous at $\rho$. It generalizes Cox's (1987) Theorem 4 in several respects: notably among them, we use a weaker topology on the space of preference profiles, and we drop his "limited asymmetry" assumption. With the result of Banks, Duggan, and Le Breton (2002) that the support of mixed strategy equilibria of the electoral game lie in the uncovered set, we have upper hemicontinuity of the mixed strategy equilibrium correspondence at profiles where the set of pure strategy equilibria is non-empty, i.e., $K[\rho] \neq \emptyset$. 
Proposition 25 Assume $A$ is a compact metric space; $\Omega$ is a complete and separable metric

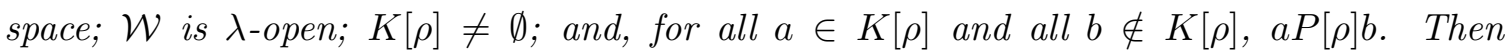
$U C[\rho]=U D[\rho]=K[\rho]$ and $U C[\cdot]$ is upper hemicontinuous at $\rho$.

Proof: That $U C[\rho]=K[\rho]$ is immediate. Suppose that $U C[\cdot]$ is not upper hemicontinuous at $\rho$, so that there exists an open set $V \supseteq U C[\rho]$ such that, for all open neighborhoods $U$ of $\rho$, there exists some $\rho_{U} \in U$ such that $U C\left[\rho_{U}\right] \backslash V \neq \emptyset$. Since the space of profiles is a metric space, we may define $U_{n}$ as the open ball around $\rho$ with radius $1 / n$, and we let $\rho_{n}=\rho_{U_{n}}$. Of course, $\left\{\rho_{n}\right\} \rightarrow \rho$. For each $\rho_{n}$, let $a_{n} \in U C\left[\rho_{n}\right] \backslash V$. Since $A$ is compact, we may assume with loss of generality that $\left\{a_{n}\right\}$ converges to some $a \in A \backslash V$. Therefore, $a \notin U C[\rho]=K[\rho]$. By assumption, for any $b^{*} \in K[\rho]$, we have $b^{*} P[\rho] a$. We claim that there is some $m$ such that $b^{*} C\left[\rho_{m}\right] a_{m}$, a contradiction.

By Lemma 6, there exists $m$ such that for all $n \geq m, b^{*} P\left[\rho_{n}\right] a_{n}$. Suppose that, for all $m$, there exists $n \geq m$ and $b_{n} \in P\left[\rho_{n}\right]\left(b^{*}\right) \backslash P\left[\rho_{n}\right]\left(a_{n}\right)$. Let $\left\{b_{n_{k}}\right\}$ be a convergent subsequence, with limit $b$. Since $a R\left[\rho_{n_{k}}\right] b_{n_{k}}$ for all $k$, Lemma 6 implies $a R[\rho] b$. By our assumption of external stability, $b \notin K[\rho]$ and $b^{*} P[\rho] b$. By Lemma 6 again, $b^{*} P\left[\rho_{n_{k}}\right] b_{n_{k}}$ for $k$ large enough, a contradiction. Therefore, there exists $m$ such that, for all $n \geq m, P\left[\rho_{n}\right]\left(b^{*}\right) \subseteq P\left[\rho_{n}\right]\left(a_{n}\right)$.

Suppose now that, for all $m$, there exists $n \geq m$ and $c_{n} \in R\left[\rho_{n}\right]\left(b^{*}\right) \backslash R\left[\rho_{n}\right]\left(a_{n}\right)$. Let $\left\{c_{n_{k}}\right\}$ be a convergent subsequence, with limit $c$. By Lemma $6, c R[\rho] b^{*}$ which implies $c \in K[\rho]$ and $c P[\rho] a$. By Lemma 6 again, $c_{n_{k}} P\left[\rho_{n_{k}}\right] a_{n_{k}}$ for $k$ large enough, a contradiction. Therefore, there exists $m$ such that, for all $n \geq m, R\left[\rho_{n}\right]\left(b^{*}\right) \subseteq R\left[\rho_{n}\right]\left(a_{n}\right)$. Therefore, $b^{*} C\left[\rho_{m}\right] a_{m}$ for high enough, completing the proof.

Conditions for external stability of the core used here are given in Propositions 1 and 17. Under these conditions, $U C[\rho]$ coincides with $K[\rho]$ and is actually a singleton. Because the uncovered set is non-empty for all profiles under the assumptions of Proposition 25, the proposition implies that $U C[\cdot]$ is actually continuous at such profiles.

Proposition 25 bears on the analysis of the uncovered set for large, finite electorates, examined in the spatial modelling literature, ${ }^{18}$ where the uncovered set is conjectured to "shrink" with probability one to a centrally located point under majority voting. Indeed, suppose that a countably infinite set of voters $i=1,2, \ldots$ have preferences given by a continuous mapping $r: \Re^{k} \rightarrow \mathcal{R}$, where $A$ is assumed to be a compact metric space and $\Re^{k}$ is a space of preference parameters, denoted $\gamma$. Here, $r(\gamma)$ is a voter's weak preference relation at $\gamma$, with $p(\gamma)$ the corresponding strict preference. Let $F$ be a continuous distribution over $\Re^{k}$, and for now assume that admits a "central" alternative: there exists $a^{*} \in A$ such that, for all $b \in A$, we have $\mu_{F}\left(\left\{\gamma: a^{*} p(\gamma) b\right\}\right)>1 / 2$, where $\mu_{F}$ is the Borel probability measure generated by $F .{ }^{19}$ Thus, assuming $\mathcal{W}$ is majority rule, $a^{*}$ would be an externally stable core point if the distribution of preferences were given by $F$. Suppose that the preferences of voters $i=1,2, \ldots$ are drawn independently from the distribution $F$, and let $U C\left[\gamma_{1}, \ldots, \gamma_{n}\right]$

\footnotetext{
${ }^{18}$ See Feld, Grofman, and Miller (1988) and Tovey (1992).

${ }^{19}$ If each $r(\gamma)$ were Euclidean, then $a^{*}$ would be a median of $F$ in all directions.
} 
be the uncovered set generated by majority rule applied to any finite draw of preference parameters.

We claim that, with probability one, $U C\left[\gamma_{1}, \ldots, \gamma_{n}\right]$ converges in a strong sense (the Hausdorff metric) to $a^{*}$ as the number of voters goes to infinity. This can be proved as follows. Taking any sequence of draws $\gamma_{1}, \gamma_{2}, \ldots$ for which the empirical distribution converges weakly to $F$, Skorokhod's Theorem (see Hildenbrand's (1974) result 37) yields a measure space $(\Omega, \Sigma, \lambda)$ and measurable mappings $\phi_{n}, \phi: \Omega \rightarrow \Re^{k}$ such that (i) for each $n, \lambda \circ \phi_{n}^{-1}$ matches the empirical distribution of preference parameters for the electorate of size $n$, (ii) $\lambda \circ \phi^{-1}=\mu_{F}$, and (iii) for $\lambda$-almost every $\omega, \phi_{n}(\omega) \rightarrow \phi(\omega)$. Therefore, majority preferences and the uncovered set for this electorate at profile $\rho_{n}=r_{n} \circ \phi_{n}$ are identical to those of the finite electorate. Furthermore, $a^{*}$ is the core at profile $\rho=r \circ \phi$ and $\Delta\left(\rho_{n}, \rho\right) \rightarrow$ 0 , and convergence of $U C\left[\gamma_{1}, \ldots, \gamma_{n}\right]$ to $a^{*}$ then follows by applying Proposition 25 to the sequence $\left\{\rho^{n}\right\}$. By the Glivenko-Cantelli Theorem (Billingsley (1995), Theorem 20.6), the preceding observations hold for almost every sequence of parameters, as claimed. In fact, Proposition 25 delivers more: the same qualitative conclusion holds if the distribution of characteristics is close, in the weak* topology, to admitting a central alternative.

The assumption that $A$ is compact in Proposition 25 is needed, unless voter preferences are restricted. To see this, suppose $X=\Re$ and there is just one voter, whose preferences are represented by the utility function $u(a)=-|a|$. Consider a sequence of preferences represented by the utility function

$$
u_{n}(a)= \begin{cases}-|a| & \text { if } a \leq \frac{n}{2} \\ \frac{n}{2}-2|a-n| & \text { if } a>\frac{n}{2}\end{cases}
$$

At the original preference relation, the core and uncovered set are $\{0\}$, while the core and uncovered set are $\{n\}$ along the sequence: closed convergence is not strong enough to rule out such approximations. This example obviously violates convexity of voter preferences.

In fact, the upper hemicontinuity result of Proposition 25 can be proved without compactness of $A$, if we restrict attention to the space of profiles of convex voter preferences. The proof of the proposition, with that restriction, is easily modified using the following lemma, which provides a compact bound on the uncovered set in a neighborhood of profiles of convex voter preferences. We omit the statement and proof of this variant of Proposition 25 . When $A$ is a subset of a vector space, we let $\mathbf{P}^{c}$ denote the set of profiles $\rho$ such that $\lambda(\{\omega \in \Omega: \rho(\omega)$ is convex $\})=1$.

Lemma 7 Assume $A$ is a convex subset of $\Re^{n}$ with the relative topology; $\Omega$ is a complete and separable metric space; for all $a \in A, \lambda(\{\omega \in \Omega: \rho(\omega)(a)$ is compact $\})=1$; and $\mathcal{W}$ is proper and $\lambda$-open. Let $\rho \in \mathbf{P}^{c}$. There exist an open subset $U \subseteq \mathbf{P}$ with $\rho \in U$ and a compact subset $Y \subseteq A$ such that, for all $\rho^{\prime} \in U \cap \mathbf{P}^{c}, U C\left[\rho^{\prime}\right] \subseteq Y$.

Proposition 25 states that upper hemicontinuity of $U C[\cdot]$ holds at $\rho$ whenever, among other things, the core at $\rho$ is non-empty. The correspondence $U C[\cdot]$ is not generally upper hemicontinuous when the core is empty, as demonstrated by the following example. Let $A$ 


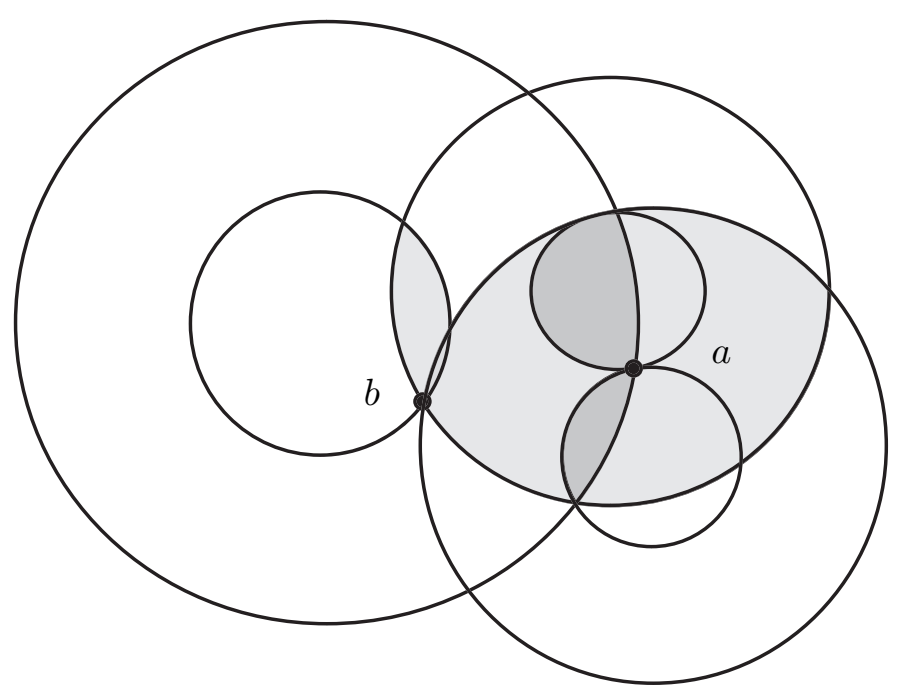

Figure 2: Alternative $a$ covers $b$

be a subset of $\Re^{2}$; let $\Omega=\{1,2,3\}$, where $\lambda$ is uniform; assume preferences $\left(R_{1}, R_{2}, R_{3}\right)$ are strictly convex, with indifference contours depicted in Figure 2; and assume $\mathcal{W}$ is majority rule. For this electorate, $a C b$ and $b \notin U C$. Perturbing preferences as depicted in Figure 3 , $a$ now fails to cover $b$, and we can specify preferences so that, for arbitrarily small perturbations, $b$ is uncovered, violating upper hemicontinuity.

Finally, we turn to lower hemicontinuity of the undominated set. For the next proposition, assume $\Omega$ is a topological space, and let $\mathbf{P}^{*}$ be the subset of profiles $\rho$ such that

- $\rho$ is continuous,

- for all $a \in A, \lambda(\{\omega \in \Omega: \rho(\omega)(a)$ is compact $\})=1$,

- $\lambda(\{\omega \in \Omega: \rho(\omega)$ is convex $\})=1$,

- LSWP holds at $\rho$,

- for all $a \in A, R[\rho](a)=\overline{P[\rho](a)} \cup\{a\}$,

- for all $a \in A, R^{-1}[\rho](a)=\overline{P^{-1}[\rho](a)} \cup\{a\}$,

- for all $a, b \in A$, if $P[\rho](a)=P[\rho](b)$ and $R[\rho](a)=R[\rho](b)$, then $a=b$.

Note that Proposition 17 gives sufficient conditions, involving dispersion of voter preferences, for the fifth requirement above, and Proposition 19 gives sufficient conditions for the sixth. While we do not give sufficient conditions for the last requirement, we conjecture that it is fairly unrestrictive in multidimensional spaces. Thus, $\mathbf{P}^{*}$ should not be too "sparse." 20

\footnotetext{
${ }^{20}$ The last restriction in the definition of $\mathbf{P}^{*}$ is not vacuous, however. It is violated for majority rule with an odd number of voters with Euclidean preferences that admit a nonempty core: then majority preferences are transitive and coincide with the core voter's. We suspect that violations of our condition, as with this example, are non-generic in multiple dimensions.
} 


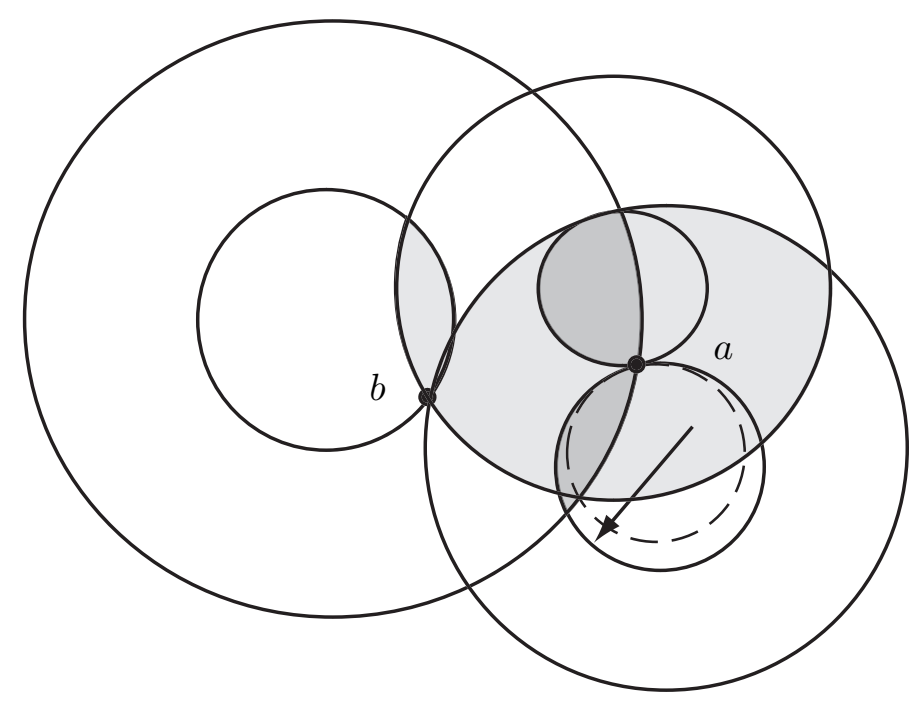

Figure 3: Alternative $b$ is uncovered

Proposition 26 Assume $A$ is a convex subset of $\Re^{n}$ with the relative topology; $\Omega$ is a compact metric space; and $\mathcal{W}$ is proper and $\lambda$-open. Then $U D[\cdot]$ is lower hemicontinuous on $\mathbf{P}^{*}$.

The proof of this result is contained in the appendix. As a metric space, the set of alternatives in the last proposition is paracompact (Aliprantis and Border (1999), Theorem 2.86), so Michael's Selection Theorem (Aliprantis and Border (1999), Theorem 16.61) yields a continuous selection from the correspondence with values equal to the closed, convex hull of $U D[\rho]$. Thus, the closed, convex hull of $U C[\rho]$ also admits a continuous selection. Such a selection result may be of interest if this correspondence is being used to predict outcomes in the second stage of a decision process: it may be, for example, that party members nominate candidates based on outcomes in a later general election stage, and that they use the closed, convex hull of the uncovered set as a solution for the second stage. In such examples, the existence of a continuous selection would be desirable for analyzing equilibria in the first stage.

If voters in the first stage have probabilistic beliefs about second-stage outcomes, then it may be appropriate to use the set of probability measures over undominated alternatives as a solution for the second stage, in which case convexity is automatic. Let $U D^{*}[\rho]$ denote the closure of the set of Borel probability measures $\mu$ on $A$ such that $\mu(U D[\rho])=1$. If $U D[\cdot]$ is lower hemicontinuous, then, with the weak* topology on the space of probability measures, $U D^{*}[\cdot]$ is also lower hemicontinuous. The latter correspondence clearly has closed, convex values. While the space of probability measures is not a Banach space, so Michael's Selection Theorem cannot be applied, the selection result relies only on a metric vector space structure, local convexity, and completeness. By Repovš and Semenov's (1998) Theorem $(1.5)^{*}$, the "probabilistic undominated set" $U D^{*}[\cdot]$ does indeed have a continuous selection, as would the "probabilistic" version of the uncovered set. 


\section{Conclusion}

We have analyzed social choice in a general version of the spatial model, encompassing the usual Euclidean setting, but also environments with a finite set of alternatives (as in the literature on tournaments) and environments with infinite-dimensional spaces of alternatives. Because we have replaced the typical strict convexity assumption with a weaker restriction on shared weak preferences, we also capture most economic environments, where agents' preferences are strictly convex in their own consumption only. We have proposed a generalization of simple games to infinite electorates, allowing us to describe the structure of power in society quite generally. Our results synthesize and generalize a number of existing results, and they extend the "institution-free" analysis of social choice to infinite electorates. We give a primitive analysis of properties of social preferences, providing sufficient conditions for continuity of social preferences and a new dispersion condition on voter preferences that delivers thin social indifference curves. We establish the generic emptiness of the core. We provide general conditions for existence of uncovered and undominated alternatives, and we examine the continuity properties of those sets.

\section{A Appendix}

\section{A.1 Maximal Elements}

Given a set $X$, with elements $x, y, z$, etc., an element $x$ is $Q$-maximal in $Y \subseteq X$ if $x \in Y$ and, for all $y \in Y, y Q x$ implies $x Q y$. We say $x$ is $Q$-maximal if it is $Q$-maximal in $X$. We define upper and lower sections of $Q$ as above. We say $Q$ is upper semicontinuous if $Q(x)$ is closed for all $x$. The next proposition gives conditions under which the set of $Q$-maximal elements is non-empty.

Proposition A1 If $Q$ is transitive and upper semicontinuous, and if $Q(x)$ is nonempty and compact for some $x$, then $Q(x)$ contains a $Q$-maximal element.

Proof: Take any $Q$-chain, $E$, in $Q(x)$. If $Q(y)=\emptyset$ for any $y \in E$, then $y$ is $Q$-maximal. Otherwise, by transitivity and upper semicontintuity, $\{Q(y): y \in E\}$ is a collection of compact subsets of $Q(x)$ with the finite intersection property, so there exists $z \in \bigcap_{y \in E} Q(y)$. Thus, $z$ is a $Q$-upper bound for $E$ in $Q(x)$. By Zorn's lemma, $Q$ has a maximal element, say $x^{*}$, in $Q(x)$. If $x^{*}$ is not maximal in $X$, then there exists $w \in X$ such that $w Q x^{*}$ and not $x^{*} Q w$. By transitivity, $w Q x^{*} Q y$ implies $w \in Q(y)$, however, a contradiction.

If we strengthen our compactness assumption in Proposition A1, we can deduce the external stability of the $Q$-maximal elements.

Proposition A2 Assume $Q$ is transitive and upper semicontinuous, and $Q(x)$ is compact for all $x$. If $x$ is not $Q$-maximal, then there exists $Q$-maximal $x^{*}$ such that $x^{*} Q x$ and not $x Q x^{*}$. 
Proof: Take any $x$ that is not $Q$-maximal, so $Q(x) \neq \emptyset$. Since $Q(x)$ is compact, it contains a $Q$-maximal element, say $x^{*}$. Thus, $x^{*} Q x$. Suppose $x Q x^{*}$. Since $x$ is not $Q$-maximal, there exists $y$ such that $y Q x$ and not $x Q y$. By transitivity, $y Q x Q x^{*}$ implies $y Q x^{*}$. Since $x^{*}$ is $Q$-maximal, we have $x^{*} Q y$. By transitivity, however, $x Q x^{*} Q y$ implies $x Q y$, a contradiction. Therefore, not $x Q x^{*}$.

Define the relation $Q^{*}$ as follows: $x Q^{*} y$ if and only if $Q(x) \subseteq Q(y)$. Note that $Q^{*}$ is reflexive and transitive. The next proposition shows that upper semicontinuity of $Q$ is inherited by $Q^{*}$, when the initial relation $Q$ has lower hemicontinuous upper sections.

Proposition A3 If $Q$ is upper semicontinuous, and if $Q(\cdot)$ is lower hemicontinuous as a correspondence, then $Q^{*}$ is upper semicontinuous.

Proof: Take any $x$ and any net $\left\{x_{\alpha}\right\}$ in $Q^{*}(x)$ converging to some $y$. Take any $z \in Q(y)$, and suppose $z \notin Q(x)$. By upper semicontinuity, $G=X \backslash Q(x)$ is open. Of course, $Q(y) \cap G \neq \emptyset$. By lower hemicontinuity, there exists $\alpha$ such that $Q\left(x_{\alpha}\right) \cap G \neq \emptyset$, but then $Q\left(x_{\alpha}\right) \nsubseteq Q(x)$, a contradiction. Therefore, $z \in Q(x)$, and we conclude $y \in Q^{*}(x)$, as desired.

Another sufficient condition for upper semicontinuity of $Q^{*}$ is open lower sections of the initial relation.

Proposition A4 If $Q^{-1}(x)$ is open for all $x$, then $Q^{*}$ is upper semicontinuous.

Proof: Take any $x$ and a net $\left\{x_{\alpha}\right\}$ in $Q^{*}(x)$ with $x_{\alpha} \rightarrow y$. We need to show $y Q^{*} x$, i.e., $Q(y) \subseteq Q(x)$. Take any $z \in Q(y)$, i.e., $y \in Q^{-1}(z)$. Since $Q^{-1}(z)$ is open, there exists $\alpha^{\prime}$ such that, for all $\alpha \geq \alpha^{\prime}, x_{\alpha} \in Q^{-1}(z)$, i.e., $z \in Q\left(x_{\alpha}\right)$. Since $x_{\alpha} Q^{*} x$, we have $z \in Q(x)$, as desired.

Let $\mathcal{Q}$ denote the set of closed relations on $X$, endowed with the topology of closed convergence. Let $\Theta$ be a topological space, and consider a mapping $Q: \Theta \rightarrow \mathcal{Q}$, and values $Q[\theta]$. We say the mapping $Q[\cdot]$ is outer continuous if, for all nets $\left\{\theta_{\alpha}\right\}$ in $\Theta$ converging to some $\theta$ and $\left\{\left(x_{\alpha}, y_{\alpha}\right)\right\}$ in $X \times X$ converging to some $(x, y), x_{\alpha} Q\left[\theta_{\alpha}\right] y_{\alpha}$ for all $\alpha$ implies $x Q[\theta] y$. Let $U Q[\theta]$ denote the $Q[\theta]$-maximal elements. We say the correspondence $U Q[\cdot]: \Theta \rightarrow \rightarrow$ $X$ has locally relatively compact range if, for every $\theta \in \Theta$, there exists an open subset $U \subseteq \Theta$ with $\theta \in U$ and a compact subset $Y \subseteq X$ such that, for all $\theta^{\prime} \in U, U Q\left[\theta^{\prime}\right] \subseteq Y$. The next proposition provides conditions for lower hemi-continuity of the maximal element correspondence.

Proposition A5 Assume that, for all $\theta \in \Theta, Q[\theta]$ is anti-symmetric, $U Q[\theta] \neq \emptyset$, and, for all $x \notin U Q[\theta]$, there exists $y \in U Q[\theta]$ such that $y Q x ; Q[\cdot]$ is outer continuous; and $U Q[\cdot]$ has locally relatively compact range. Then $U Q[\cdot]$ is lower hemi-continuous. 
Proof: Take any $\theta \in \Theta$, and let $G$ be any open subset $G \subseteq X$ such that $G \cap U Q[\theta] \neq \emptyset$. Let $x \in G \cap U Q[\theta]$. Let $\left\{\theta_{\alpha}\right\}$ be a net converging to $\theta$, and suppose that, for each $\alpha$, $U Q\left[\theta_{\alpha}\right] \cap G=\emptyset$. Thus, for each $\alpha, x \notin U Q\left[\theta_{\alpha}\right]$. By external stability, there exists $y_{\alpha} \in$ $U Q\left[\theta_{\alpha}\right]$ such that $y_{\alpha} Q\left[\theta_{\alpha}\right] x$. Moreover, $y_{\alpha} \notin G$ for each $\alpha$. Since $U Q[\cdot]$ has locally relatively compact range, $\left\{y_{\alpha}\right\}$ has a convergent subnet with limit, say, $y$. By outer continuity, $y Q[\theta] x$. Because $y_{\alpha} \notin G$ for all $\alpha$, we have $y \neq x$, and then anti-symmetry implies not $x Q[\theta] y$, which implies $x \notin U Q[\theta]$, a contradiction.

Now consider a mapping $R: \Theta \rightarrow \mathcal{Q}$ with values $R[\theta]$. Following the above convention, write $R[\theta](x)$ for the upper section of $R[\theta]$ at $x$, and say $x R^{*}[\theta] y$ if and only if $R[\theta](x) \subseteq$ $R[\theta](y)$. Write $x P[\theta] y$ if and only if not $x R[\theta] y$.

Proposition A6 Assume that, for all $\theta \in \Theta, R[\theta]$ is complete and, for all $x \in X$, $R[\theta](x)=\overline{P[\theta](x)} \cup\{x\}$; and $R[\cdot]$ is outer continuous. Then $R^{*}[\cdot]$ is outer continuous.

Proof: Take any nets $\left\{\theta_{\alpha}\right\}$ converging to $\theta$ and $\left\{\left(x_{\alpha}, y_{\alpha}\right)\right\}$ converging to $(x, y)$ such that, for each $\alpha, x_{\alpha} R^{*}\left[\theta_{\alpha}\right] y_{\alpha}$. Take any $z \in R[\theta](x)$. Suppose $z \notin R[\theta](y)$. Then, because $R[\theta](y)$ is closed, $G=P^{-1}[\theta](y)$ is an open set containing $z$. Case 1: $z=x$. Since $y P[\theta] x$, outer continuity implies that, for some subnet, also indexed by $\alpha, y_{\alpha} P\left[\theta_{\alpha}\right] x_{\alpha}$. But, by reflexivity of $R\left[\theta_{\alpha}\right]$ and $x_{\alpha} R^{*}\left[\theta_{\alpha}\right] y_{\alpha}$, we have $x_{\alpha} \in R\left[\theta_{\alpha}\right]\left(x_{\alpha}\right) \subseteq R\left[\theta_{\alpha}\right]\left(y_{\alpha}\right)$, a contradiction. Case 2: $z \neq x$. Since $z \in \overline{P[\theta](x)}$, there exists $w \in P[\theta](x) \cap G$, so $y P[\theta] w$. It then follows from outer continuity that, for some subnet, also indexed by $\alpha, y_{\alpha} P\left[\theta_{\alpha}\right] w$ for all $\alpha$. Then, by $x_{\alpha} R^{*}\left[\theta_{\alpha}\right] y_{\alpha}$, we have $x_{\alpha} P\left[\theta_{\alpha}\right] w$ for all $\alpha$. By completeness of $R\left[\theta_{\alpha}\right]$, this implies $x_{\alpha} R\left[\theta_{\alpha}\right] w$ for all $\alpha$, and outer continuity implies $x R[\theta] w$, a contradiction. Therefore, $z \in R[\theta](y)$, and we conclude that $x R^{*}[\theta] y$.

\section{A.2 Omitted Proofs of Propositions}

We use the terminology and results from the previous subsection to provide proofs for propositions where they were omitted from the text.

Proposition 2 Assume $R(a)$ is compact for some $a \in A$, and $R(b)$ is closed for all $b \in A$. Then $U C \neq \emptyset$.

Proof: Since $R(b)$ is closed, it follows that $P^{-1}(b)=X \backslash R(b)$ is open for all $b \in A$, and Proposition A4 implies that $P^{*}$ is upper semicontinuous. By irreflexivity of $P$, we have $P^{*}(a) \subseteq R(a)$, and it follows that $P^{*}(a)$ is compact. Applying Proposition A1, there exists a $P^{*}$-maximal element, which must belong to $U C$. Therefore, $U C \neq \emptyset$.

Proposition 3 Assume $R(a)$ is compact for some $a \in A, R(b)$ is closed for all $b \in A$, and $R(\cdot)$ is lower hemicontinuous as a correspondence. Then $U D \neq \emptyset$. 
Proof: As in the proof of Proposition 2, $P^{-1}(b)$ is open for all $b \in A$, and Proposition A4 implies that $P^{*}$ is upper semicontinuous. By Proposition A3, $R^{*}$ is upper semicontinuous as well. Thus, $P^{*} \cap R^{*}$ is upper semicontinuous and is clearly transitive. Furthermore, $R^{*}(a) \subseteq R(a)$ by reflexivity of $R$, so $R^{*}(a)$ is compact. Applying Proposition A1 yields a $\left(P^{*} \cap R^{*}\right)$-maximal element, which must belong to $U D$. Therefore, $U D \neq \emptyset$.

Proposition 4 Assume $R(a)$ is compact for all $a \in A$ and $R(\cdot)$ is lower hemicontinuous as a correspondence.

1. If $a \notin U D$, then there exists $b \in U D$ such that $b D a$.

2. If $a \notin U C$, then there exists $b \in U C$ such that $b C a$.

Proof: By the argument in the proof of Proposition 3, it follows that $R^{*}(a)$, and therefore $\left(P^{*} \cap R^{*}\right)(a)$, is compact for all $a \in A$. Take $a$ and $c$ such that $c D a$, implying $c\left(P^{*} \cap R^{*}\right) a$. If $c \in U D$, then we set $b=c$. If $c \notin U D$, then, by Proposition A2, there exists a $\left(P^{*} \cap R^{*}\right)$ maximal element $b$, therefore belonging to $U D$, such that $b\left(P^{*} \cap R^{*}\right) c$. Therefore, $P(b) \subseteq$ $P(c) \subseteq P(a)$ and $R(b) \subseteq R(c) \subseteq R(a)$. Since $c D a$, we have $b D a$, and external stability of $U D$ follows. Now let $c \in A$ be such that $c C a$. If $c \in U D$, then $c \in U C$, and we are done. If $c \notin U D$, then, by the first part of the proposition, there is some undominated, hence uncovered, $b$ such that $b D c$. Then $b D c C a$ implies $b C a$.

Proposition 26 Assume $A$ is a convex subset of $\Re^{n}$ with the relative topology; $\Omega$ is a compact metric space; and $\mathcal{W}$ is proper and $\lambda$-open. Then $U D[\cdot]$ is lower hemicontinuous on $\mathbf{P}^{*}$.

Proof: To use Proposition A6, let $\Theta=\mathbf{P}^{*}$. By Proposition 11, since $\mathcal{W}$ is proper, it follows that $R[\rho]$ is complete. The condition $R[\rho](a)=\overline{P[\rho](a)} \cup\{a\}$ for all $\rho \in \mathbf{P}^{*}$, holds by assumption. By Lemma $6, R[\cdot]$ is outer continuous on $\mathbf{P}^{*}$, so, by Proposition A6, $R^{*}[\cdot]$ is outer continuous. To see that $R^{*}[\rho]$ is anti-symmetric for all $\rho \in \mathbf{P}^{*}$, suppose $a R^{*}[\rho] b$, i.e., $R(a) \subseteq R(b)$. By assumption, we have $R^{-1}[\rho](a)=\overline{P^{-1}[\rho](a)} \cup\{a\}$ and $R^{-1}[\rho](b)=\overline{P^{-1}[\rho](b)} \cup\{b\}$. From Lemma 2, this implies $P[\rho](a) \cup\{a\}=R[\rho](a)^{\circ} \cup\{a\}$ and $P[\rho](b) \cup\{b\}=R[\rho](b)^{\circ} \cup\{b\}$, so Lemma 1 yields $P[\rho](a) \subseteq P[\rho](b)$. Then our assumptions on $\mathbf{P}^{*}$ imply $a=b$, as claimed. By Proposition $12, R[\rho]$ is upper semicontinuous, and $R[\rho](\cdot)$ is lower hemicontinuous by Proposition 13, so Proposition A3 implies that $R^{*}[\rho]$ is upper semicontinuous. Propositions 14 and 15 imply that $R[\rho](a)$ is compact for all $a \in A$, and since $R^{*}(a) \subseteq R(a)$, it follows that $R^{*}(a)$ is compact for all $a \in A$. Propositions A1 and A2 then imply non-emptiness and external stability of the $R^{*}[\rho]$-maximal elements, denoted $U R^{*}[\rho]$.

To see that $U R^{*}[\cdot]$ has locally relatively compact range, note that, for every $\rho \in \mathbf{P}^{*}$, Lemma 7 yields an open set $U \subseteq \mathbf{P}^{*}$ with $\rho \in U$ and a compact set $Y$ such that, for all $\rho^{\prime} \in U$, 
$U C\left[\rho^{\prime}\right] \subseteq Y$. We claim that, for all $\rho \in \mathbf{P}^{*}, U R^{*}[\rho] \subseteq U D[\rho]$, which implies $U R^{*}[\rho] \subseteq U C[\rho]$. Take $a \in U R^{*}[\rho]$, and suppose there exists $b \in A$ such that $b D[\rho] a$, i.e., $R[\rho](b) \subseteq R[\rho](a)$ and $P[\rho](b) \subseteq P[\rho](a)$, at least one inclusion strict. In fact, because $\rho \in \mathbf{P}^{*}$, the first must be strict, but then $a \notin U R^{*}[\rho]$, a contradiction. Therefore, $U R^{*}[\rho] \subseteq U D[\rho]$, and it follows that $U R^{*}[\cdot]$ has locally relatively compact range. Proposition A5 then implies that $U R^{*}[\cdot]$ is lower hemicontinuous on $\mathbf{P}^{*}$.

Finally, we claim that $U D[\rho] \subseteq U R^{*}[\rho]$ for all $\rho \in \mathbf{P}^{*}$. Suppose $a \notin U R^{*}[\rho]$, so there is some $b \in A$ such that $b R^{*}[\rho] a$ and not $a R^{*}[\rho] b$. Note that $b \neq a$. By definition, $R[\rho](b) \subseteq$ $R[\rho](a)$. By Lemmas 1 and 2, we also have $P[\rho](b) \subseteq P[\rho](a)$. At least one inclusion must be strict, for otherwise $\rho \in \mathbf{P}^{*}$ implies $a=b$. Therefore, $b D[\rho] a$, implying $a \notin U D[\rho]$, a contradiction. This establishes the claim, and it follows that $U D[\cdot]=U R^{*}[\cdot]$ on $\mathbf{P}^{*}$. Thus, $U D[\cdot]$ is lower hemicontinuous on $\mathbf{P}^{*}$.

\section{A.3 Proofs of Lemmas}

\section{Lemma 1}

1. If $R(a)=\overline{P(a)} \cup\{a\}$ and $R(b)=\overline{P(b)} \cup\{b\}$, then $P(a) \subseteq P(b)$ implies $R(a) \subseteq R(b)$.

2. If $P(a) \cup\{a\}=R(a)^{\circ} \cup\{a\}$ and $P(b) \cup\{b\}=R(b)^{\circ} \cup\{b\}$, then $R(a) \subseteq R(b)$ implies $P(a) \subseteq P(b)$.

Proof: To prove the first part of the proposition, suppose $P(a) \subseteq P(b)$. Clearly, $\overline{P(a)} \cup$ $\{a\} \subseteq \overline{P(b)} \cup\{a\}$. Note that $b \notin P(a)$ by irreflexivity of $P$, so $a \in R(b)$. Therefore,

$$
R(a)=\overline{P(a)} \cup\{a\} \subseteq \overline{P(b)} \cup\{a\} \subseteq R(b),
$$

as required. To prove the second part, suppose $R(a) \subseteq R(b)$. Then

$$
P(a) \cup\{a\}=R(a)^{\circ} \cup\{a\} \subseteq R(b)^{\circ} \cup\{a, b\}=P(b) \cup\{a\} .
$$

Since $a \notin P(a)$ by irreflexivity of $P, P(a) \subseteq P(b)$, as required.

Lemma 2 Assume $A$ is Hausdorff. Then $P(a) \cup\{a\}=R(a)^{\circ} \cup\{a\}$ for all $a \in A$ if and only if $R^{-1}(a)=\overline{P^{-1}(a)} \cup\{a\}$ for all $a \in A$.

Proof: First assume $P(a) \cup\{a\}=R(a)^{\circ} \cup\{a\}$ for all $a \in A$. Since $A$ is Hausdorff, the singleton $\{a\}$ is closed, and since $a \notin P(a)$, by irreflexivity, we see that

$$
P(a)=[P(a) \cup\{a\}] \backslash\{a\}=\left[R(a)^{\circ} \cup\{a\}\right] \backslash\{a\}
$$

is open: if $a \notin R(a)^{\circ}$, then $\left[R(a)^{\circ} \cup\{a\}\right] \backslash\{a\}=R(a)^{\circ}$ is open; if $a \in R(a)^{\circ}$, then $\left[R(a)^{\circ} \cup\{a\}\right] \backslash\{a\}=R(a)^{\circ} \backslash\{a\}$ is the intersection of two open sets and is open. Therefore, 
$R^{-1}(a)$ is closed. Note that $b \in P^{-1}(a)$ is equivalent to $a \in P(b)$, which holds only if $a \in R(b)^{\circ}$, which implies $b \in R^{-1}(a)$. Thus, $P^{-1}(a) \subseteq R^{-1}(a)$. Since $R$ is reflexive and $R^{-1}(a)$ is closed, we then have $\overline{P^{-1}(a)} \cup\{a\} \subseteq R^{-1}(a)$. Now take any $b \in R^{-1}(a)$ such that $b \neq a$. Suppose that $b \notin \overline{P^{-1}(a)}$, i.e., $b \in R(a)^{\circ}$. Since $b \neq a$, we then have $b \in P(a)$, a contradiction. Therefore, $b \in \overline{P^{-1}(a)}$, and we have shown $R^{-1}(a) \subseteq \overline{P^{-1}(a)} \cup\{a\}$, as required.

Now assume $R^{-1}(a)=\overline{P^{-1}(a)} \cup\{a\}$ for all $a \in A$. Since $\{a\}$ is closed, it follows that $R^{-1}(a)$ is closed, and therefore $P(a)$ is open. Note that $b \in P(a)$ implies $a \in P^{-1}(b) \subseteq$ $R^{-1}(b)$, so $b \in R(a)$. Thus, $P(a) \subseteq R(a)$. Since $P(a)$ is open, we then have $P(a) \cup\{a\} \subseteq$ $R(a)^{\circ} \cup\{a\}$. Now take any $b \in R(a)^{\circ}$ such that $b \neq a$. Suppose $b \notin P(a)$, i.e., $b \in R^{-1}(a)$. We then have $b \in \overline{P^{-1}(a)}$, contradicting $b \in R(a)^{\circ}$. Therefore, $b \in P(a)$, and we have shown $R(a)^{\circ} \cup\{a\} \subseteq P(a) \cup\{a\}$, as required.

Lemma 3 Assume $R$ is complete and $P$ is asymmetric.

1. If $a \in P^{2}(b)$, then $P(b) \nsubseteq P(a)$ and $R(b) \nsubseteq R(a)$.

2. If $P(b) \nsubseteq P(a)$ or $R(b) \nsubseteq R(a)$, then $a \in R^{2}(b)$.

3. If $R^{-1}(a)=\overline{P^{-1}(a)} \cup\{a\}$ for all $a \in A$, then $a \in P^{2}(b)$ if and only if $P(b) \nsubseteq P(a)$.

4. If $R(a)=\overline{P(a)} \cup\{a\}$ for all $a \in A$, then $a \in P^{2}(b)$ if and only if $R(b) \nsubseteq R R(a)$.

Proof: To prove the first part of the lemma, suppose $a \in P^{2}(b)$, so either $a P b$ or there exists $c \in A$ such that $a P c P b$. In the first case, $b \in R(b) \backslash R(a)$. In the second case, by completeness, we have $c \in R(b) \backslash R(a)$. In both cases, $R(b) \nsubseteq R(a)$ and, by asymmetry of $P$, $P(b) \nsubseteq P(a)$. To prove the second part of the lemma, note that $P(b) \nsubseteq P(a)$ means $a R c P b$ for some $c \in A$, and that $R(b) \nsubseteq R(a)$ means $a P c R b$. In both cases, by completeness, $a \in R^{2}(b)$. To prove the third part of the lemma, note that one direction follows from part 1. For the other direction, suppose $P(b) \nsubseteq P(a)$, so that there exists $c \in A$ such that $a R c P b$. If $c=a$, then the argument is finished. Otherwise, $c \in \overline{P^{-1}(a)}$. Since $c \in P(b)$, an open set, there exists $d \in P^{-1}(a) \cap P(b)$, as required. To prove the fourth part of the lemma, note that one direction follows from part 1. For the other direction, suppose $R(b) \nsubseteq R(a)$, so there exists $c \in A$ such that $a P c R b$. If $c=b$, then the argument is finished. Otherwise, $c \in \overline{P(b)}$. Since $c \in P^{-1}(a)$, an open set, there exists $d \in P^{-1}(a) \cap P(b)$, as required.

Lemma 4 Assume $A$ is Hausdorff, second countable, and locally compact; $\Omega$ is a compact topological space and $\rho$ is continuous; and LSWP holds. Let $\mathcal{S}$ be an arbitrary simple game. Then $\bigcup_{S \in \mathcal{S}} P_{S}(\cdot)$ is lower hemicontinuous as a correspondence.

Proof: Take any $a \in A$ and any open set $G \subseteq A$ such that $G \cap \bigcup_{S \in \mathcal{S}} P_{S}(a) \neq \emptyset$, i.e., $b \in G \cap \bigcup_{S \in \mathcal{S}} P_{S}(a)$ for some $b \in A$. Thus, $\{\omega \in \Omega: b \pi(\omega) a\} \in \mathcal{S}$, and therefore $S=$ 
$\{\omega \in \Omega: b \rho(\omega) a\} \in \mathcal{S}$. Since $\rho$ is continuous, $S$ is closed. Since $S^{*}$ has $\lambda$-measure one, $T=S \cap S^{*} \in \mathcal{S}$. Since $\Omega$ is compact, and since $S$ and $S^{*}$ are closed, $T$ is compact. By LSWP, there exists a sequence $\left\{c_{n}\right\}$ in $P_{T}(a)$ such that $c_{n} \rightarrow b$. Mas-Colell (1977) establishes the existence of a jointly continuous function $U: \mathcal{R} \times A \rightarrow \Re$ such that, for all $R \in \mathcal{R}$, $U(R, \cdot)$ is a utility representation of $R$. By construction, $U\left(\rho(\omega), c_{n}\right)-U(\rho(\omega), a)>0$ for all $\omega \in T$. Since this function is continuous in $\omega$ and $T$ is compact, it follows that $\min _{\omega \in T} U\left(\rho(\omega), c_{n}\right)-U(\rho(\omega), a)>0$. By continuity, there is an open set $X_{n} \subseteq A$ with $a \in X_{n}$ such that $\min _{\omega \in T} U\left(\rho(\omega), c_{n}\right)-U(\rho(\omega), x)>0$ for all $x \in X_{n}$. Picking $n$ high enough, we have $c_{n} \in G$ and, by the preceding discussion, $c_{n} \in \bigcup_{S \in \mathcal{S}} P_{S}(x)$ for all $x \in X_{n}$, implying $G \cap \bigcup_{S \in \mathcal{S}} P_{S}(x) \neq \emptyset$ over an open set around $a$, as required.

Lemma 5 Assume $A$ is Hausdorff, second countable, and locally compact; $\Omega$ is a topological space; and $\rho$ is continuous. Let $S \in \Sigma$ be compact. Then $P_{S}$ is open.

Proof: Let $U: \mathcal{R} \times A \rightarrow \Re$ be a jointly continuous function such that, for all $R \in \mathcal{R}$, $U(R, \cdot)$ is a utility representation of $R$. Take $(a, b) \in P_{S}$, so $U(\rho(\omega), a)>U(\rho(\omega), b)$ for all $\omega \in S$. By continuity and compactness, $\min _{\omega \in S} U(\rho(\omega), a)-U(\rho(\omega), b)>0$. By continuity, there exists an open set $G \subseteq A \times A$ such that $\min _{\omega \in S} U(\rho(\omega), c)-U(\rho(\omega), d)>0$ for all $(c, d) \in G$.

Lemma 6 Assume $A$ is a locally compact, complete, separable metric space; $\Omega$ is a complete and separable metric space; and $\mathcal{W}$ is $\lambda$-open. Let $\rho_{n} \rightarrow \rho$, let $a_{n} \rightarrow a$, and let $b_{n} \rightarrow b$. If $a_{n} R\left[\rho_{n}\right] b_{n}$ for all $n$, then $a R[\rho] b$.

Proof: From the Polish version of Lusin's Theorem (Aliprantis and Border (1999), Theorem 10.8), for all $\epsilon>0$, there exists a compact subset $Y_{\epsilon}$ of $\Omega$ such that $\lambda\left(Y_{\epsilon}\right) \geq 1-\epsilon$ and the profile $\rho$, restricted to $Y_{\epsilon}$, is continuous. Since $\tilde{d}\left(\rho_{n}, \rho\right) \rightarrow 0$ in the $L_{1}$-norm, Aliprantis and Border's (1999) Theorem 12.6 yields a subsequence $\left\{\rho_{n_{k}}\right\}$ such that $\tilde{d}\left(\rho_{n_{k}}, \rho\right) \rightarrow 0 \lambda$-almost surely. Now let $U: \mathcal{R} \times A \rightarrow \Re$ be a jointly continuous mapping such that, for all $R \in \mathcal{R}$, $U(R, \cdot)$ is a utility representation of $R$. For all $\omega \in \Omega$ and all $k$, define

$$
\begin{aligned}
& \Phi_{k}^{a}(\omega)=U\left(\rho_{n_{k}}(\omega), a_{n_{k}}\right) \\
& \Phi_{k}^{b}(\omega)=U\left(\rho_{n_{k}}(\omega), b_{n_{k}}\right)
\end{aligned} \quad \text { and } \quad \begin{aligned}
& \Phi^{a}(\omega)=U(\rho(\omega), a) \\
& \Phi^{b}(\omega)=U(\rho(\omega), b) .
\end{aligned}
$$

Since $U$ is jointly continuous, we deduce that $\Phi_{k}^{a} \rightarrow \Phi^{a}$ and $\Phi_{k}^{b} \rightarrow \Phi^{b} \lambda$-almost surely. From Egoroff's Theorem (Aliprantis and Border (1999), Theorem 9.37), there are compact subsets $Y_{\epsilon}^{a}$ and $Y_{\epsilon}^{b}$ of $\Omega$ such that

- $\lambda\left(Y_{\epsilon}^{a}\right) \geq 1-\epsilon$ and $\lambda\left(Y_{\epsilon}^{b}\right) \geq 1-\epsilon$

- $\Phi_{k}^{a} \rightarrow \Phi^{a}$ uniformly on $Y_{\epsilon}^{a}$

- $\Phi_{k}^{b} \rightarrow \Phi^{b}$ uniformly on $Y_{\epsilon}^{b}$. 
Now let $S=\{\omega \in \Omega: b \pi(\omega) a\}$, and suppose that $S \in \mathcal{W}$. Since $\Omega$ is Polish, Aliprantis and Border's (1999) Theorem 10.7 implies the existence of a compact subset $Y_{\epsilon}^{\prime} \subseteq S$ such that $\lambda\left(Y_{\epsilon}^{\prime}\right) \geq \lambda(S)-\epsilon$. Let $Y_{\epsilon}^{\prime \prime}=Y_{\epsilon} \cap Y_{\epsilon}^{\prime} \cap Y_{\epsilon}^{a} \cap Y_{\epsilon}^{b}$. Since $\lambda\left(Y_{\epsilon}^{\prime \prime}\right) \geq \lambda(S)-4 \epsilon$ and $\mathcal{W}$ is $\lambda$-open, we have $Y_{\epsilon}^{\prime \prime} \in \mathcal{W}$ for small enough $\epsilon$. Furthermore, since $\rho$ is continuous on $Y_{\epsilon}^{\prime \prime}$, it follows that $\Phi^{b}-\Phi^{a}$ is continuous on $Y_{\epsilon}^{\prime \prime}$. And since $Y_{\epsilon}^{\prime \prime}$ is compact and $\Phi^{b}(\omega)-\Phi^{a}(\omega)>0$ for all $\omega \in Y_{\epsilon}^{\prime \prime}$, there exists $\delta>0$ such that,

$$
\Phi^{b}(\omega)-\Phi^{a}(\omega)>\delta \text { for all } \omega \in Y_{\epsilon}^{\prime \prime}
$$

But by uniform convergence on $Y_{\epsilon}^{a}$ and $Y_{\epsilon}^{b}$, we deduce that, for $k$ large enough,

$$
\left|\Phi_{k}^{a}(\omega)-\Phi^{a}(\omega)\right| \leq \frac{\delta}{2} \text { for all } \omega \in Y_{\epsilon}^{\prime \prime}
$$

and

$$
\left|\Phi_{k}^{b}(\omega)-\Phi^{b}(\omega)\right| \leq \frac{\delta}{2} \text { for all } \omega \in Y_{\epsilon}^{\prime \prime}
$$

Therefore, for $k$ large enough,

$$
\Phi_{k}^{b}(\omega)-\Phi_{k}^{a}(\omega)>0 \text { for all } \omega \in Y_{\epsilon}^{\prime \prime} .
$$

Since $Y_{\epsilon}^{\prime \prime} \in \mathcal{W}$, this implies that, for $k$ large enough, $b_{n_{k}} P\left[\rho_{n_{k}}\right] a_{n_{k}}$, a contradiction. Therefore, $S \notin \mathcal{W}$, implying $a R[\rho] b$, as required.

Lemma 7 Assume $A$ is a convex subset of $\Re^{n}$ with the relative topology; $\Omega$ is a complete and separable metric space; for all $a \in A, \lambda(\{\omega \in \Omega: \rho(\omega)(a)$ is compact $\})=1$; and $\mathcal{W}$ is proper and $\lambda$-open. Let $\rho \in \mathbf{P}^{c}$. There exist an open subset $U \subseteq \mathbf{P}$ with $\rho \in U$ and a compact subset $Y \subseteq A$ such that, for all $\rho^{\prime} \in U \cap \mathbf{P}^{c}, U C\left[\rho^{\prime}\right] \subseteq Y$.

Proof: Take any $a \in A$. By Proposition 16, $R[\rho](a)$ is compact. We use the notation $B_{r}(a) \subseteq N$ to denote the closed ball of radius $r$ centered at $a$. Let $r_{1}$ satisfy $R[\rho](a) \subseteq$ $B_{r_{1}}(a)$. By Proposition 16, then $Y_{1}=R[\rho]\left(B_{2 r_{1}}(a)\right)$ is also compact. Let $r_{2}$ satisfy $Y_{1} \subseteq$ $B_{r_{2}}(a)$, and define the compact set $Y_{2}=B_{2 r_{2}}(a)$. Suppose there does not exist an open subset $U$ of $\mathbf{P}$ such that, for all $\rho^{\prime} \in U \cap \mathbf{P}^{c}, U C\left[\rho^{\prime}\right] \subseteq Y_{2}$. Since the space of profiles is a metric space, we may define $U_{n}$ as the open ball around $\rho$ with radius $1 / n$. For each $n$, let $\rho_{n} \in U_{n} \cap \mathbf{P}^{c}$ and $b_{n} \in U C\left[\rho_{n}\right] \backslash Y_{2}$. Of course, $\rho_{n} \rightarrow \rho$. Since $\mathcal{W}$ is proper, Proposition 11 implies that $P\left[\rho_{n}\right]$ is asymmetric, and by Proposition 6 , there exists $c_{n} \in A$ satisfying $b_{n} R\left[\rho_{n}\right] c_{n} R\left[\rho_{n}\right] a$. In particular, $c_{n} \in R\left[\rho_{n}\right](a)$.

We claim that, for high enough $n$, we have $c_{n} \in Y_{1}$. If not, then there is a subsequence $\left\{c_{n_{k}}\right\}$ such that $c_{n_{k}} \notin Y_{1}$ for all $k$. Define $d_{k}$ as

$$
d_{k}=a+\frac{2 r_{1}}{\left\|c_{n_{k}}-a\right\|}\left(c_{n_{k}}-a\right)
$$


so that $\left\|d_{k}-a\right\|=2 r_{1}$ for all $k$. Let $S_{k}=\left\{\omega \in \Omega: c_{n_{k}} \rho_{n_{k}}(\omega) a\right\} \in \mathcal{B}$ be the set of voters who weakly prefer $c_{n_{k}}$ to $a$ at $\rho_{n_{k}}$, let $S_{k}^{\prime}=\left\{\omega \in \Omega: \rho_{n_{k}}(\omega)\right.$ is convex $\}$, and let $T_{k}=S_{k} \cap S_{k}^{\prime}$. By assumption, $T_{k} \in \mathcal{B}$. By convexity, we have $d_{k} \rho_{n_{k}}(\omega) a$ for all $\omega \in T_{k}$, which implies $d_{k} R\left[\rho_{n_{k}}\right] a$. Since $d_{k} \in B_{2 r_{1}}(a)$ for all $k$, compactness of $B_{2 r_{1}}(a)$ yields a convergent subsequence, also indexed by $k$, with limit, say, $d$. By continuity of the Euclidean norm, we have $\|d-a\|=2 r_{1}$, which implies $d \notin B_{r_{1}}(a)$, which implies $d \notin R[\rho](a)$. By Lemma 6 , however, $\rho_{n_{k}} \rightarrow \rho, d_{k} \rightarrow d$, and $d_{k} R\left[\rho_{n_{k}}\right] a$ for all $k$ imply that $d R[\rho] a$, a contradiction. Therefore, for high enough $n$, we have $c_{n} \in Y_{1}$.

We now claim that, for high enough $n$, we also have $b_{n} \in Y_{2}$. If not, then there is a subsequence $\left\{b_{n_{k}}\right\}$ such that $b_{n_{k}} \notin Y_{2}$ for all $k$. Assume $k$ sufficiently high that $c_{n_{k}} \in Y_{1}$, and define $e_{k}=c_{n_{k}}+\alpha_{k}\left(b_{n_{k}}-c_{n_{k}}\right)$, where

$$
\alpha_{k}=\max \left\{\alpha \in[0,1]: c_{n_{k}}+\alpha\left(b_{n_{k}}-c_{n_{k}}\right) \in Y_{2}\right\}
$$

Note that this is well-defined, since $c_{n_{k}} \in Y_{1} \subseteq B_{r_{2}}(a) \subseteq Y_{2}$, and note that $\left\|e_{k}-a\right\|=2 r_{2}$ for all $k$. Let $S_{k}=\left\{\omega \in \Omega: b_{n_{k}} \rho_{n_{k}}(\omega) c_{n_{k}}\right\} \in \mathcal{B}$ be the set of voters who weakly prefer $b_{n_{k}}$ to $c_{n_{k}}$ at $\rho_{n_{k}}$, let $S_{k}^{\prime}=\left\{\omega \in \Omega: \rho_{n_{k}}(\omega)\right.$ is convex $\}$, and let $T_{k}=S_{k} \cap S_{k}^{\prime}$. By convexity, we have $e_{k} \rho_{n_{k}}(\omega) c_{n_{k}}$ for all $\omega \in T_{k}$, which implies $d_{k} R\left[\rho_{n_{k}}\right] c_{n_{k}}$. Since $\left(c_{n_{k}}, e_{k}\right) \in Y_{1} \times Y_{2}$ for all $k$, there is a convergent subsequence, also indexed by $k$, with limit, say, $(c, e)$. Then, of course, $c \in Y_{1}$. By continuity of the norm, we have $\|e-a\|=2 r_{2}$, which implies $e \notin B_{r_{2}}(a)$, which implies $e \notin R[\rho]\left(B_{2 r_{1}}(a)\right)$, which implies $e \notin R[\rho](c)$. By Lemma 6, however, $\rho_{n_{k}} \rightarrow \rho$, $e_{k} \rightarrow e, c_{n_{k}} \rightarrow c$, and $e_{k} R\left[\rho_{n_{k}}\right] c_{n_{k}}$ for all $k$ imply that $e R[\rho] c$, a contradiction. Therefore, for high enough $n$, we have $b_{n} \in Y_{2}$, a contradiction. We conclude that there exists an open subset $U$ of $\mathbf{P}$ such that, for all $\rho^{\prime} \in U \cap \mathbf{P}^{c}, U C\left[\rho^{\prime}\right] \subseteq Y_{2}$.

\section{References}

[1] C. Aliprantis and K. Border (1999) Infinite Dimensional Analysis: A Hitchhiker's Guide, New York: Springer-Verlag.

[2] T. Armstrong (1980) "Arrow's Theorem with Restricted Coalition Algebras," Journal of Mathematical Economics, 7, 55-75.

[3] D. Austen-Smith and J. Banks (1999) Positive Political Theory I: Collective Preference, Ann Arbor: University of Michigan Press.

[4] K. Arrow (1963) Social Choice and Individual Values, 2nd edition, New Haven: Yale University Press.

[5] J. Banks (1995) "Singularity Theory and Core Existence in the Spatial Model," Journal of Mathematical Economics, 24, 523-536.

[6] J. Banks and J. Duggan (1999) "A Bargaining Model of Collective Choice," Caltech Social Science Working Paper no. 1053. 
[7] J. Banks and J. Duggan (2000) "A Bargaining Model of Collective Choice," American Political Science Review, 94, 73-88.

[8] J. Banks, J. Duggan, and M. Le Breton (2002) "Bounds for Mixed Strategy Equilibria and the Spatial Model of Elections," Journal of Economic Theory, 103, 88-105.

[9] P. Billingsley (1995) Probability and Measure, 3rd Edition, New York: John Wiley and Sons.

[10] G. Bordes, M. Le Breton, and M. Salles (1992) "Gillies and Miller's Subrelations of a Relation over an Infinite Set of Alternatives: General Results and Applications to Voting Games," Mathematics of Operations Research, 17, 509-518.

[11] A. Caplin and B. Nalebuff (1988) "On 64\% Majority Voting," Econometrica, 56, 787814.

[12] G. Cox (1984) "Non-Collegial Simple Games and the Nowhere Denseness of the Set of Preference Profiles having a Core," Social Choice and Welfare, 1, 159-164.

[13] G. Cox (1987) "The Uncovered Set and the Core," American Journal of Political Science, 31, 408-422.

[14] O. Davis and M. Hinich (1966) "A Mathematical Model of Policy Formation in a Democratic Society," in Mathematical Applications in Political Science, II, J. Bernd, Ed., Dallas: Southern Methodist University Press.

[15] O. Davis, M. Degroot, and M. Hinich (1972) "Social Preference Orderings and Majority Rule," Econometrica, 40, 147-157.

[16] G. Debreu (1964) "Continuity Properties of Paretian Utility," International Economic Review, 5, 285-293.

[17] A. Downs (1957) An Economic Theory of Democracy, New York: Harper and Row.

[18] J. Duggan (2002) "Equilibrium Existence in Discontinuous Zero-sum Games with Applications to Spatial Models of Elections," mimeo., University of Rochester.

[19] J. Duggan and M. Jackson (2004) "Mixed Strategy Equilibrium and Deep Covering in Multidimensional Electoral Competition," mimeo.

[20] J. Duggan and M. Le Breton (2001) "Mixed Refinements of Shapley's Saddles and Weak Tournaments," Social Choice and Welfare, 18, 65-78.

[21] B. Dutta and J.-F. Laslier (1999) "Comparison Functions and Choice Correspondences," Social Choice and Welfare, 16, 513-532.

[22] R. Farquharson (1969) The Theory of Voting, New Haven: Yale University Press. 
[23] S. Feld, B. Grofman, and N. Miller (1988) "Centripetal Forces in Spatial Voting: On the Size of the Yolk," Public Choice, 59, 37-50.

[24] S. Feld, B. Grofman, R. Hartley, M. Kilgour, and N. Miller (1987) "The Uncovered Set in Spatial Voting Games," Theory and Decision, 23, 129-155.

[25] M. Fey (2002) "May's Theorem with an Infinite Population," Social Choice and Welfare, forthcoming.

[26] P. Fishburn (1970) "Arrow's Impossibility Theorem: Concise Proof and Infinite Voters," Journal of Economic Theory, 2, 103-106.

[27] P. Fishburn (1977) "Condorcet Social Choice Functions," SIAM Journal of Applied Mathematics, 33, 469-489.

[28] D. Fisher and J. Ryan (19912) "Optimal Strategies for a Generalized 'Scissors, Paper, and Stone' Game," American Mathematical Monthly, 99, 935-942.

[29] A. Gomberg, C. Martinelli, and R. Torres (2002) "Anonymity in Large Societies," mimeo., Instituto Tecnológico Autónomo de México.

[30] J. Grandmont (1978) "Intermediate Preferences and Majority Rule," Econometrica, 46, 317-330.

[31] P. Halmos (1960) Naive Set Theory, Springer-Verlag.

[32] R. Hartley and M. Kilgour (1987) "The Geometry of the Uncovered Set," Mathematical Social Sciences, 14, 175-183.

[33] W. Hildenbrand (1974) Core and Equilibria of a Large Economy, Princeton: Princeton University Press.

[34] A. Kirman and D. Sonderman (1972) "Arrow's Theorem, Many Agents, and Invisible Dictators," Journal of Economic Theory, 5, 267-277.

[35] D. Koehler (1990) "The Size of the Yolk: Computations for Odd and Even-Numbered Committees," Social Choice and Welfare, 7, 231-245.

[36] G. Kramer (1972) "Sophisticated Voting over Multidimensional Spaces," Journal of Mathematical Sociology, 2, 165-180.

[37] G. Kramer (1978) "Existence of an Electoral Equilibrium," in Game Theory and Political Science, P. Ordeshook, Ed., New York: New York University Press.

[38] G. Laffond, J.-F. Laslier, and M. Le Breton (1993) "The Bipartisan Set of a Tournament Game," Games and Economic Behavior, 5, 182-201.

[39] J.-F. Laslier (1997) Tournament Solutions and Majority Voting, New York: Springer. 
[40] M. Le Breton (1987) "On the Core of Voting Games," Social Choice and Welfare, 4, 295-305.

[41] A. Mas-Colell (1977) "On the Continuous Representation of Preorders," International Economic Review, 18, 509-513.

[42] R. McKelvey (1976) "Intransitivities in Multi-dimensional Voting Models and some Implications for Agenda Control," Journal of Economic Theory, 12, 472-482.

[43] R. McKelvey (1979) "General Conditions for Global Intransitivities in Formal Voting Models," Econometrica, 47, 1086-1112.

[44] R. McKelvey (1986) "Covering, Dominance, and Institution-free Properties of Social Choice," American Journal of Political Science, 30, 283-314.

[45] R. McKelvey, P. Ordeshook, and P. Ungar (1980) "Conditions for Voting Equilibria in Continuous Voter Distributions," SIAM Journal of Applied Mathematics, 39, 161-168.

[46] N. Miller (1980) "A New Solution Set for Tournaments and Majority Voting: Further Graph-theoretical Approaches to the Theory of Voting," American Journal of Political Science, 24, 68-96.

[47] H. Moulin (1986) "Choosing from a Tournament," Social Choice and Welfare, 3, 271291.

[48] R. Myerson (1993) "Incentives to Cultivate Favored Minorities Under Alternative Electoral Systems," American Political Science Review, 87, 856-869.

[49] P. Ordeshook (1993) "The Spatial Analysis of Elections and Committees: Four Decades of Research," California Institute of Technology, mimeo.

[50] E. Penn (2002) "Alternate Definitions of the Uncovered Set and Their Implications," mimeo., California Institute of Technology.

[51] J. Peris and B. Subiza (1999) "Condorcet Choice Correspondences for Weak Tournaments," Social Choice and Welfare, 16, 217-231.

[52] C. Plott (1967) "A Notion of Equilibrium and its Possibility under Majority Rule," American Economic Review, 57, 787-806.

[53] D. Repovš and P. Semenov (1998) Continuous Selections of Multivalued Mappings, Boston: Kluwer Academic Publishers.

[54] W. Riker (1980) "Implications from the Disequilibrium of Majority Rule for the Study of Institutions," American Political Science Review, 74, 432-446.

[55] A. Rubinstein (1979) "A Note on the Nowhere Denseness of Societies having an Equilibrium under Majority Rule," Econometrica, 47, 511-514. 
[56] D. Saari (1997) "The Generic Existence of a Core for q-Rules," Economic Theory, 9, 219-260.

[57] N. Schofield (1983) "Generic Instability of Majority Rule," Review of Economic Studies, 50, 695-705.

[58] L. Shapley (1962) "Simple Games: An Outline of the Descriptive Theory," Behavioral Science, 7, 59-66.

[59] K. Shepsle and B. Weingast (1984) "Uncovered Sets and Sophisticated Voting Outcomes with Implications for Agenda Institutions," American Journal of Political Science, 28, 49-74.

[60] R. Sikorski (1964) Boolean Algebras, 2nd ed., New York: Springer-Verlag.

[61] C. Tovey (1992) "The Almost Surely Shrinking Yolk," mimeo.

[62] G. Tullock (1967) "The General Irrelevance of the General Possibility Theorem," Quarterly Journal of Economics, 81, 256-270. 